\title{
The eigenvalues of the sample covariance matrix of a multivariate heavy-tailed stochastic volatility model
}

\author{
ANJA JANSSEN ${ }^{1, *}$, THOMAS MIKOSCH${ }^{1, * *, \dagger}$, \\ MOHSEN REZAPOUR ${ }^{2}$ and XIAOLEI XIE ${ }^{1, 末}$ \\ ${ }^{1}$ Department of Mathematical Sciences, University of Copenhagen, Universitetsparken 5, DK-2100 Copen- \\ hagen, Denmark.E-mail: *anja@math.ku.dk, ${ }^{* *}$ mikosch@math.ku.dk, url: ${ }^{\dagger} w w w . m a t h . k u . d k / \sim m i k o s c h$, \\ ¥xie@math.ku.dk \\ ${ }^{2}$ Department of Statistics, Faculty of Mathematics and Computer, Shahid Bahonar University of Kerman, \\ Kerman,Iran.E-mail: mohsenrzp@gmail.com
}

\begin{abstract}
We consider a multivariate heavy-tailed stochastic volatility model and analyze the large-sample behavior of its sample covariance matrix. We study the limiting behavior of its entries in the infinite-variance case and derive results for the ordered eigenvalues and corresponding eigenvectors. Essentially, we consider two different cases where the tail behavior either stems from the i.i.d. innovations of the process or from its volatility sequence. In both cases, we make use of a large deviations technique for regularly varying time series to derive multivariate $\alpha$-stable limit distributions of the sample covariance matrix. For the case of heavy-tailed innovations, we show that the limiting behavior resembles that of completely independent observations. In contrast to this, for a heavy-tailed volatility sequence the possible limiting behavior is more diverse and allows for dependencies in the limiting distributions which are determined by the structure of the underlying volatility sequence.
\end{abstract}

Keywords: dependent entries; eigenvectors; largest eigenvalues; regular variation; sample covariance matrix; stochastic volatility

\section{Introduction}

\subsection{Background and motivation}

The study of sample covariance matrices is fundamental for the analysis of dependence in multivariate time series. Besides from providing estimators for variances and covariances of the observations (in case of their existence), the sample covariance matrices are a starting point for dimension reduction methods like principal component analysis. Accordingly, the special structure of sample covariance matrices and their largest eigenvalues has been intensively studied in random matrix theory, starting with i.i.d. Gaussian observations and more recently extending results to more general light-tailed distributions which satisfy some moment assumptions like in the four moment theorem of Tao and $\mathrm{Vu}$ [47].

However, with respect to the analysis of financial time series, such a moment assumption is often not suitable. Instead, in this work, we will analyze the large sample behavior of sample 
covariance matrices under the assumption that the marginal distributions of our observations are regularly varying with index $\alpha<4$ which implies that fourth moments do not exist and the behavior of the largest eigenvectors differs significantly from the light-tailed case; see, for example, [3] and [25]. In this case, we would expect the largest eigenvalues of the sample covariance matrix to inherit heavy-tailed behavior as well; see, for example, Ben Arous and Guionnet [8], Belinschi et al. [6], Bordenave and Guionnet [10], Auffinger et al. [2], Soshnikov [45,46], Davis et al. [17], Heiny and Mikosch [31] for the case of i.i.d. entries. Furthermore, in the context of financial time series we have to allow for dependencies both over time and between different components and indeed it is often the aim of the analysis to discover and test for these dependencies. The sample covariance matrix is an important tool for this and has for example been analyzed in Plerou et al. [41] and Davis et al. [23,24]. The detection of dependencies among assets also plays a crucial role in portfolio optimization based on multi-factor pricing models, where principal component analysis is one way to derive the main driving factors of a portfolio; cf. Campbell et al. [12] and recent work by Lam and Yao [37].

The literature on the asymptotic behavior of sample covariance matrices derived from dependent heavy-tailed data is, however, relatively sparse up till now. Starting with the analysis of the sample autocorrelation of univariate linear heavy-tailed time series in Davis and Resnick [15,16], the theory has recently been extended to multivariate heavy-tailed time series with linear structure in Davis et al. [23,24], cf. also the recent survey article by Davis et al. [17]. But most of the standard models for financial time series such as GARCH and stochastic volatility models are non-linear. In this paper, we will therefore focus on a class of multivariate stochastic volatility models of the form

$$
X_{i t}=\sigma_{i t} Z_{i t}, \quad t \in \mathbb{Z}, 1 \leq i \leq p,
$$

where $\left(Z_{i t}\right)$ is an i.i.d. random field independent of a strictly stationary ergodic field $\left(\sigma_{i t}\right)$ of nonnegative random variables; see Section 2 for further details. Stochastic volatility models have been studied in detail in the financial time series literature; see, for example, Andersen et al. [1], Part II. They are among the simplest models allowing for conditional heteroscedasticity of a time series. In view of independence between the $Z$ - and $\sigma$-fields, dependence conditions on $\left(X_{i t}\right)$ are imposed only via the stochastic volatility $\left(\sigma_{i t}\right)$. Often it is assumed that $\left(\log \sigma_{i t}\right)$ has a linear structure, which provides a tractable and flexible class of models while ensuring positivity of the volatility process; see Davis and Mikosch [21]. This modelling goes back to Taylor [48], who first introduced the so-called log-normal stochastic volatility model where the log-volatility process is assumed to be a Gaussian AR(1) process.

In this paper, we are interested in the case when the marginal and finite-dimensional distributions of $\left(X_{i t}\right)$ have power-law tails. Due to independence between $\left(\sigma_{i t}\right)$ and $\left(Z_{i t}\right)$, heavy tails of $\left(X_{i t}\right)$ can be due to the $Z$ - or the $\sigma$-field. Here we will consider two cases: (1) the tails of $Z$ dominate the right tail of $\sigma$ and (2) the right tail of $\sigma$ dominates the tail of $Z$. The third case when both $\sigma$ and $Z$ have heavy tails and are tail-equivalent will not be considered in this paper. Case (1) is typically more simple to handle; see Davis and Mikosch [19-21] for extreme value theory, point process convergence and central limit theory with infinite variance stable limits. Case (2) is more subtle as regards the tails of the finite-dimensional distributions. The literature on stochastic volatility models with a heavy-tailed volatility sequence is so far sparse but the 
interest in these models has been growing recently; see Mikosch and Rezapour [38], Kulik and Soulier [36] and Janßen and Drees [34]. In particular, it has been shown that these models offer a lot of flexibility with regard to the extremal dependence structure of the time series, ranging from asymptotic dependence of consecutive observations (cf. [38]) to asymptotic independence of varying degrees (cf. [36] and [34]).

\subsection{Aims, main results and structure}

After introducing the general model in Section 2, we first deal with the case of heavy-tailed innovations and a light-tailed volatility sequence in Section 3. The first step in our analysis is to describe the extremal structure of the corresponding process by deriving its so-called tail process; see Section 2.3 and Proposition 3.1. This allows one to apply an infinite variance stable central limit theorem from Mikosch and Wintenberger [39] (see Appendix A) to derive the joint limiting behavior of the entries of the sample covariance matrix of this model. This leads to the main results in the first case: Theorems 3.3 and 3.6. They say, roughly speaking, that all values on the off-diagonals of the sample covariance matrix are negligible compared to the values on the diagonals. Furthermore, the values on the diagonal converge, under suitable normalization, to independent $\alpha$-stable random variables, so the limiting behavior of this class of stochastic volatility models is quite similar to the case of i.i.d. heavy-tailed random variables. This fairly tractable structure allows us also to derive explicit results about the asymptotic behavior of the ordered eigenvalues and corresponding eigenvectors which can be found in Sections 3.3 and 3.4. In particular, we will see that in this model the eigenvectors are basically the unit canonical basis vectors which describe a very weak form of extremal dependence. With a view towards portfolio analysis, our assumptions imply that large movements of the market are mainly driven by one single asset, where each asset is equally likely to be this extreme driving force.

In the second case of a heavy-tailed volatility sequence combined with light-tailed innovations, which we analyze in Section 4, we see that the range of possible limiting behaviors of the entries of the sample covariance matrix is more diverse and depends on the specific structure of the underlying volatility process. We make the common assumption that our volatility process is log-linear, where we distinguish between two different cases for the corresponding innovation distribution of this process. Again, for both cases, we first derive the specific form of the corresponding tail process (see Proposition 4.4) which then allows us to derive the limiting behavior of the sample covariance matrix entries, leading to the main results in the second case: Theorems 4.6 and 4.10. We show that the sample covariance matrix can feature non-negligible offdiagonal components, therefore clearly distinguishing from the i.i.d. case, if we assume that the innovations of the log-linear volatility process are convolution equivalent. We discuss concrete examples for both model specifications and the corresponding implications for the asymptotic behavior of ordered eigenvalues and corresponding eigenvectors at the end of Section 4.

Section 5 contains a small simulation study which illustrates our results for both cases and also includes a real-life data example for comparison. From the foreign exchange rate data that we use, it is notable that the corresponding sample covariance matrix features a relatively large gap between the largest and the second largest eigenvalue and that the eigenvector corresponding to the largest eigenvalue is fairly spread out, that is, all its components are of a similar order of 
magnitude. This implies that the model discussed in Section 3 may not be that suitable to catch the extremal dependence of this data, and that there is not one single component that is most affected by extreme movements but instead all assets are affected in a similar way. We perform simulations for three different specifications of models from Sections 3 and 4 . They illustrate that the models analyzed in Section 4 are capable of exhibiting more diverse asymptotic behaviors of the sample covariance matrix and in particular non-localized dominant eigenvectors.

Some useful results for the (joint) tail and extremal behavior of random products are gathered in Appendix B. These results may be of independent interest when studying the extremes of multivariate stochastic volatility models with possibly distinct tail indices. We mention in passing that there is great interest in non-linear models for log-returns of speculative prices when the number of assets $p$ increases with the sample size $n$. We understand our analysis as a first step in this direction.

\section{The model}

We consider a stochastic volatility model

$$
X_{i t}=\sigma_{i t} Z_{i t}, \quad i, t \in \mathbb{Z},
$$

where $\left(Z_{i t}\right)$ is an i.i.d. field independent of a strictly stationary ergodic field $\left(\sigma_{i t}\right)$ of non-negative random variables. We write $Z, \sigma, X$ for generic elements of the $Z-, \sigma$ - and $X$-fields such that $\sigma$ and $Z$ are independent. A special case appears when $\sigma>0$ is a constant: then $\left(X_{i t}\right)$ constitutes an i.i.d. field.

For the stochastic volatility model as in (1.1), we construct the multivariate time series

$$
\mathbf{X}_{t}=\left(X_{1 t}, \ldots, X_{p t}\right)^{\prime}, \quad t \in \mathbb{Z},
$$

for a given dimension $p \geq 1$. For $n \geq 1$ we write $\mathbf{X}^{n}=\operatorname{vec}\left(\left(\mathbf{X}_{t}\right)_{t=1, \ldots, n}\right)=\left(X_{i t}\right)_{i=1, \ldots p, t=1, \ldots, n}$ $\in \mathbb{R}^{p \times n}$ and consider the non-normalized sample covariance matrix

$$
\mathbf{X}^{n}\left(\mathbf{X}^{n}\right)^{\prime}=\left(S_{i j}\right)_{i, j=1, \ldots, p}, \quad S_{i j}=\sum_{t=1}^{n} X_{i t} X_{j t}, \quad S_{i}=S_{i i}
$$

\subsection{Case (1): $Z$ dominates the tail}

We assume that $Z$ is regularly varying with index $\alpha>0$, that is,

$$
\mathbb{P}(Z>x) \sim p_{+} \frac{L(x)}{x^{\alpha}} \quad \text { and } \mathbb{P}(Z<-x) \sim p_{-} \frac{L(x)}{x^{\alpha}}, \quad x \rightarrow \infty,
$$

where $p_{+}$and $p_{-}$are non-negative numbers with $p_{+}+p_{-}=1$ and $L$ is a slowly varying function (for two positive functions $f(x), g(x)$ the notation $f(x) \sim g(x), x \rightarrow \infty$, means that 
$\left.\lim _{x \rightarrow \infty} f(x) / g(x)=1\right)$. If we assume $\mathbb{E}\left[\sigma^{\alpha+\delta}\right]<\infty$ for some $\delta>0$ then, in view of a result by Breiman [11] (see also Lemma B.1), it follows that

$$
\mathbb{P}(X>x) \sim \mathbb{E}\left[\sigma^{\alpha}\right] \mathbb{P}(Z>x) \quad \text { and } \quad \mathbb{P}(X<-x) \sim \mathbb{E}\left[\sigma^{\alpha}\right] \mathbb{P}(Z<-x), \quad x \rightarrow \infty,
$$

that is, $X$ is regularly varying with index $\alpha$. Moreover, we know from a result by Embrechts and Goldie [28] that for independent copies $Z_{1}$ and $Z_{2}$ of $Z, Z_{1} Z_{2}$ is again regularly varying with index $\alpha$; cf. Lemma B.1. Therefore, using again Breiman's result under the condition that $\mathbb{E}\left[\left(\sigma_{i 0} \sigma_{j 0}\right)^{\alpha+\delta} \mathbf{1}(i \neq j)+\sigma_{i 0}^{\alpha+\delta}\right]<\infty$ for some $\delta>0$, we have

$$
\mathbb{P}\left( \pm X_{i t} X_{j t}>x\right) \sim\left\{\begin{array}{ll}
\mathbb{E}\left[\left(\sigma_{i t} \sigma_{j t}\right)^{\alpha}\right] \mathbb{P}\left( \pm Z_{i} Z_{j}>x\right), & i \neq j, \\
\mathbb{E}\left[\sigma^{\alpha}\right] \mathbb{P}\left(Z^{2}>x\right), & i=j,
\end{array} \quad x \rightarrow \infty\right.
$$

\subsection{Case (2): $\sigma$ dominates the tail}

We assume that $\sigma \geq 0$ is regularly varying with some index $\alpha>0$ : for some slowly varying function $\ell$,

$$
\mathbb{P}(\sigma>x)=x^{-\alpha} \ell(x),
$$

and $\mathbb{E}\left[|Z|^{\alpha+\delta}\right]<\infty$ for some $\delta>0$. Now the Breiman result yields

$$
\mathbb{P}(X>x) \sim \mathbb{E}\left[Z_{+}^{\alpha}\right] \mathbb{P}(\sigma>x) \quad \text { and } \quad \mathbb{P}(X<-x) \sim \mathbb{E}\left[Z_{-}^{\alpha}\right] \mathbb{P}(\sigma>x), \quad x \rightarrow \infty
$$

Since we are also interested in the tail behavior of the products $X_{i t} X_{j t}$ we need to be more precise about the joint distribution of the sequences $\left(\sigma_{i t}\right)$. We assume

$$
\sigma_{i t}=\exp \left(\sum_{k, l=-\infty}^{\infty} \psi_{k l} \eta_{i-k, t-l}\right), \quad i, t \in \mathbb{Z}
$$

where $\left(\psi_{k l}\right)$ is a field of non-negative numbers (at least one of them being positive) such that (without loss of generality) $\max _{k l} \psi_{k l}=1$ and $\left(\eta_{i t}\right)$ is an i.i.d. random field such that a generic element $\eta$ satisfies

$$
\mathbb{P}\left(\mathrm{e}^{\eta}>x\right)=x^{-\alpha} L(x)
$$

for some $\alpha>0$ and a slowly varying function $L$. We also assume $\sum_{k, l} \psi_{k l}<\infty$ to ensure absolute summability of $\log \sigma_{i t}$. A distribution of $\eta$ that fits into this scheme is, for example, the exponential distribution; cf. also Rootzén [44] for further examples and extreme value theory for linear processes of the form $\sum_{l=-\infty}^{\infty} \psi_{l} \eta_{t-l}$. 


\subsection{Regularly varying sequences}

In Sections 3.1 and 4.1, we will elaborate on the joint tail behavior of the sequences $\left(\sigma_{i t}\right),\left(X_{i t}\right)$, $\left(\sigma_{i t} \sigma_{j t}\right)$, and $\left(X_{i t} X_{j t}\right)$. We will show that, under suitable conditions, these sequences are regularly varying with positive indices.

The notion of a univariate regularly varying sequence was introduced by Davis and Hsing [18]. Its extension to the multivariate case does not represent difficulties; see Davis and Mikosch [22]. An $\mathbb{R}^{d}$-valued strictly stationary sequence $\left(\mathbf{Y}_{t}\right)$ is regularly varying with index $\gamma>0$ if each of the vectors $\left(\mathbf{Y}_{t}\right)_{t=0, \ldots, h}, h \geq 0$, is regularly varying with index $\gamma$, that is, there exist non-null Radon measures $\mu_{h}$ on $[-\infty, \infty]^{d(h+1)} \backslash\{\mathbf{0}\}$ which are homogeneous of order $-\gamma$ (i.e., $\mu_{h}(\lambda A)=\lambda^{-\gamma} \mu_{h}(A)$ for all Borel sets $A$ and $\lambda>0$ ) such that

$$
\frac{\mathbb{P}\left(x^{-1}\left(\mathbf{Y}_{t}\right)_{t=0, \ldots, h} \in \cdot\right)}{\mathbb{P}\left(\left\|\mathbf{Y}_{0}\right\|>x\right)} \stackrel{v}{\rightarrow} \mu_{h}(\cdot) .
$$

Here $\stackrel{v}{\rightarrow}$ denotes vague convergence on the Borel $\sigma$-field of $[-\infty, \infty]^{d(h+1)} \backslash\{\boldsymbol{0}\}$ and $\|\cdot\|$ denotes any given norm; see Resnick's books $[42,43]$ as general references to multivariate regular variation.

Following Basrak and Segers [5], an $\mathbb{R}^{d}$-valued strictly stationary sequence $\left(\mathbf{Y}_{t}\right)$ is regularly varying with index $\gamma>0$ if and only if there exists a sequence of $\mathbb{R}^{d}$-valued random vectors $\left(\boldsymbol{\Theta}_{h}\right)$ independent of a Pareto $(\gamma)$ random variable $Y$, i.e., $\mathbb{P}(Y>x)=x^{-\gamma}, x>1$, such that for any $k \geq 0$,

$$
\mathbb{P}\left(x^{-1}\left(\mathbf{Y}_{0}, \ldots, \mathbf{Y}_{k}\right) \in \cdot \mid\left\|\mathbf{Y}_{0}\right\|>x\right) \stackrel{w}{\rightarrow} \mathbb{P}\left(Y\left(\boldsymbol{\Theta}_{0}, \ldots, \boldsymbol{\Theta}_{k}\right) \in \cdot\right), \quad x \rightarrow \infty .
$$

We call $\left(\boldsymbol{\Theta}_{h}\right)$ the spectral tail process of $\left(\mathbf{Y}_{t}\right)$ and $\left(Y \boldsymbol{\Theta}_{h}\right)$ the tail process. We will use both defining properties (i.e., (2.9) and (2.10)) of a regularly varying sequence.

\section{Case (1): $Z$ dominates the tail}

\subsection{Regular variation of the stochastic volatility model and its product processes}

Proposition 3.1. We assume the stochastic volatility model (2.1) and that $Z$ is regularly varying with index $\alpha>0$ in the sense of (2.4).

1. If $\mathbb{E}\left[\sigma^{\alpha+\varepsilon}\right]<\infty$ for some $\varepsilon>0$, the sequence $\left(X_{i t}\right)_{t \in \mathbb{Z}}$ is regularly varying with index $\alpha$ and the corresponding spectral tail process $\left(\Theta_{h}^{i}\right)_{h \geq 1}$ vanishes.

2. For any $i \neq j$, if $\mathbb{E}\left[\left(\sigma_{i 0} \sigma_{j 0}\right)^{\alpha+\varepsilon}\right]<\infty$ for some $\varepsilon>0$, then the sequence $\left(X_{i t} X_{j t}\right)$ is regularly varying with index $\alpha$ and the corresponding spectral tail process $\left(\Theta_{h}^{i j}\right)_{h \geq 1}$ vanishes.

Remark 3.2. If $\mathbb{E}\left[\left(\sigma_{i k} \sigma_{j l}\right)^{\alpha+\varepsilon_{i k, j l}}\right]<\infty$ for some $\varepsilon_{i k, j l}>0$ and any $(i, k) \neq(j, l)$, it is also possible to show the joint regular variation of the processes $\left(X_{i t} X_{j t}\right), i \neq j$, with index $\alpha$. The description of the corresponding spectral tail process is slightly tedious. It is not needed for the purposes of this paper and therefore omitted. 
Proof. Regular variation of the marginal distributions of $\left(X_{i t}\right)$ and $\left(X_{i t} X_{j t}\right)$ follows from Breiman's result; see (2.5) and (2.6). As regards the regular variation of the finite-dimensional distributions of $\left(X_{i t}\right)$, we have for $h \geq 1$,

$$
\begin{aligned}
\mathbb{P}\left(\left|X_{i h}\right|>x|| X_{i 0} \mid>x\right) & =\frac{\mathbb{P}\left(\min \left(\left|X_{i 0}\right|,\left|X_{i h}\right|\right)>x\right)}{\mathbb{P}\left(\left|X_{i 0}\right|>x\right)} \\
& \leq \frac{\mathbb{P}\left(\max \left(\sigma_{i 0}, \sigma_{i h}\right) \min \left(\left|Z_{i 0}\right|,\left|Z_{i h}\right|\right)>x\right)}{\mathbb{P}\left(\left|X_{i 0}\right|>x\right)} \rightarrow 0, \quad x \rightarrow \infty
\end{aligned}
$$

In the last step, we used Markov's inequality together with the moment condition $\mathbb{E}\left[\sigma^{\alpha+\varepsilon}\right]<\infty$ and the fact that $\min \left(\left|Z_{i 0}\right|,\left|Z_{i h}\right|\right)$ is regularly varying with index $2 \alpha$. This means that $\Theta_{h}^{i}=0$ for $h \geq 1$.

Similarly, for $i \neq j, h \geq 1$,

$$
\begin{aligned}
& \mathbb{P}\left(\left|X_{i h} X_{j h}\right|>x|| X_{i 0} X_{j 0} \mid>x\right) \\
& \quad \leq \frac{\mathbb{P}\left(\max \left(\sigma_{i 0} \sigma_{j 0}, \sigma_{i h} \sigma_{j h}\right) \min \left(\left|Z_{i 0} Z_{j 0}\right|,\left|Z_{i h} Z_{j h}\right|\right)>x\right)}{\mathbb{P}\left(\left|X_{i 0} X_{j 0}\right|>x\right)} \rightarrow 0 .
\end{aligned}
$$

In the last step, we again used Markov's inequality, the fact that $Z_{i 0} Z_{j 0}$ is regularly varying with index $\alpha$ (see Embrechts and Goldie [28]; cf. Lemma B.1(1) below), hence $\min \left(\left|Z_{i 0} Z_{j 0}\right|\right.$, $\left.\left|Z_{i h} Z_{j h}\right|\right)$ is regularly varying with index $2 \alpha$, and the moment condition $\mathbb{E}\left[\left(\sigma_{i 0} \sigma_{j 0}\right)^{\alpha+\varepsilon}\right]<\infty$. Hence $\Theta_{h}^{i j}=0$ for $i \neq j, h \geq 1$.

\subsection{Infinite variance stable limit theory for the stochastic volatility model and its product processes}

Theorem 3.3. Consider the stochastic volatility model (2.1) and assume the following conditions:

1. $Z$ is regularly varying with index $\alpha \in(0,4) \backslash\{2\}$.

2. $\left(\left(\sigma_{i t}\right)_{t=1,2, \ldots}\right)_{i=1, \ldots, p}$ is strongly mixing with rate function $\left(\alpha_{h}\right)$ such that for some $\delta>0$,

$$
\sum_{h=0}^{\infty} \alpha_{h}^{\delta /(2+\delta)}<\infty
$$

3. The moment condition

$$
\mathbb{E}\left[\sigma^{2 \max (2+\delta, \alpha+\epsilon)}\right]<\infty
$$

holds for the same $\delta>0$ as in (3.1) and some $\epsilon>0$.

Then

$$
a_{n}^{-2}\left(S_{1}-c_{n}, \ldots, S_{p}-c_{n}\right) \stackrel{d}{\rightarrow}\left(\xi_{1, \alpha / 2}, \ldots, \xi_{p, \alpha / 2}\right)
$$


where the $S_{i}, i=1, \ldots, p$, are as in $(2.3)$ and the $\left(\xi_{i, \alpha / 2}\right)$ are i.i.d. $\alpha / 2$-stable random variables which are totally skewed to the right,

$$
c_{n}= \begin{cases}0, & \alpha \in(0,2) \\ n \mathbb{E}\left[X^{2}\right], & \alpha \in(2,4)\end{cases}
$$

and $\left(a_{n}\right)$ satisfies $n \mathbb{P}\left(|X|>a_{n}\right) \rightarrow 1$ as $n \rightarrow \infty$.

Remark 3.4. From classical limit theory (see Feller [30], Petrov [40]), we know that (3.3) holds for an i.i.d. random field $\left(X_{i t}\right)$ with regularly varying $X$ with index $\alpha \in(0,4)$. In the case $\alpha=2$ one needs the special centering $c_{n}=n \mathbb{E}\left[X^{2} \mathbf{1}\left(|X| \leq a_{n}\right)\right]$ which often leads to some additional technical difficulties. For this reason we typically exclude this case in the sequel.

Remark 3.5. It follows from standard theory that $\alpha$-mixing of $\left(\sigma_{i t}\right)$ with rate function $\left(\alpha_{h}\right)$ implies $\alpha$-mixing of $\left(X_{i t}\right)$ with rate function $\left(4 \alpha_{h}\right)$; see Davis and Mikosch [21].

Proof. Recall the definition of $\left(\mathbf{X}_{t}\right)$ from (2.2). We will verify the conditions of Theorem A.1 for $\mathbf{X}_{t}^{2}=\left(X_{i t}^{2}\right)_{i=1, \ldots, p}, t=0,1,2, \ldots$

(1) We start by verifying the regular variation condition for $\left(\mathbf{X}_{t}\right)$; see (2.10). We will determine the sequence $\left(\boldsymbol{\Theta}_{h}\right)$ corresponding to $\left(\mathbf{X}_{t}\right)$. We have for $t \geq 1$, with the max-norm $\|\cdot\|$,

$$
\begin{aligned}
\mathbb{P}\left(\left\|\mathbf{X}_{t}\right\|>x \mid\left\|\mathbf{X}_{0}\right\|>x\right) & \leq \frac{\mathbb{P}\left(\left\|\mathbf{X}_{t}\right\|>x, \bigcup_{i=1}^{p}\left\{\left|X_{i 0}\right|>x\right\}\right)}{\mathbb{P}\left(\left\|\mathbf{X}_{0}\right\|>x\right)} \\
& \leq \sum_{i=1}^{p} \frac{\mathbb{P}\left(\left\|\mathbf{X}_{t}\right\|>x,\left|X_{i 0}\right|>x\right)}{\mathbb{P}\left(\left\|\mathbf{X}_{0}\right\|>x\right)} \\
& \leq \sum_{i=1}^{p} \sum_{j=1}^{p} \frac{\mathbb{P}\left(\left|X_{j t}\right|>x,\left|X_{i 0}\right|>x\right)}{\mathbb{P}(|X|>x)} \\
& \leq \sum_{i=1}^{p} \sum_{j=1}^{p} \frac{\mathbb{P}\left(\max \left(\sigma_{j t}, \sigma_{i 0}\right) \min \left(\left|Z_{j t}\right|,\left|Z_{i 0}\right|\right)>x\right)}{\mathbb{P}(\sigma|Z|>x)} .
\end{aligned}
$$

We observe that by Breiman's result and in view of the moment condition (3.2), for $t \geq 1$ and some positive constant $c$,

$$
\frac{\mathbb{P}\left(\max \left(\sigma_{j t}, \sigma_{i 0}\right) \min \left(\left|Z_{j t}\right|,\left|Z_{i 0}\right|\right)>x\right)}{\mathbb{P}(\sigma|Z|>x)} \sim c \frac{\mathbb{P}\left(\min \left(\left|Z_{j t}\right|,\left|Z_{i 0}\right|\right)>x\right)}{\mathbb{P}(|Z|>x)},
$$

and the right-hand side converges to zero as $x \rightarrow \infty$. We conclude that $\boldsymbol{\Theta}_{h}=\mathbf{0}$ for $h \geq 1$. We also have for $i \neq j$,

$$
\frac{\mathbb{P}\left(\left|X_{i 0}\right|>x,\left|X_{j 0}\right|>x\right)}{\mathbb{P}(|X|>x)} \leq \frac{\mathbb{P}\left(\max \left(\sigma_{i 0}, \sigma_{j 0}\right) \min \left(\left|Z_{i 0}\right|,\left|Z_{j 0}\right|\right)>x\right)}{\mathbb{P}(\sigma|Z|>x)} \rightarrow 0, \quad x \rightarrow \infty .
$$


Then, in a similar way, one can show

$$
\mathbb{P}\left(\mathbf{X}_{0} /\left\|\mathbf{X}_{0}\right\| \in \cdot \mid\left\|\mathbf{X}_{0}\right\|>x\right) \stackrel{w}{\rightarrow} \mathbb{P}\left(\boldsymbol{\Theta}_{0} \in \cdot\right)=\frac{1}{p} \sum_{i=1}^{p}\left(p_{+} \varepsilon_{\mathbf{e}_{i}}(\cdot)+p_{-} \varepsilon_{-\mathbf{e}_{i}}(\cdot)\right),
$$

where $\mathbf{e}_{i}$ are the canonical basis vectors in $\mathbb{R}^{p}, \varepsilon_{\mathbf{x}}$ is Dirac measure at $\mathbf{x}$ and $p_{ \pm}$are the tail balance factors in (2.4).

We conclude that the spectral tail process $\left(\boldsymbol{\Theta}_{h}^{(2)}\right)$ of $\left(\mathbf{X}_{t}^{2}\right)$ is given by $\boldsymbol{\Theta}_{h}^{(2)}=\mathbf{0}$ for $h \geq 1$ and from (3.5) we also have

$$
\mathbb{P}\left(\boldsymbol{\Theta}_{0}^{(2)} \in \cdot\right)=\frac{1}{p} \sum_{i=1}^{p} \varepsilon_{\mathbf{e}_{i}}(\cdot) .
$$

In particular, the condition $\sum_{i=1}^{\infty} \mathbb{E}\left[\left\|\Theta_{i}^{(2)}\right\|\right]<\infty$ in Theorem A.1(4) is trivially satisfied.

(2) Next, we want to prove the mixing condition (A.1) for the sequence $\left(\mathbf{X}_{t}^{2}\right)$. We start by observing that there are integer sequences $\left(l_{n}\right)$ and $\left(m_{n}\right)$ such that $k_{n} \alpha_{l_{n}} \rightarrow 0, l_{n}=o\left(m_{n}\right)$ and $m_{n}=o(n)$. Then we also have for any $\gamma>0$,

$$
k_{n} \mathbb{P}\left(\sum_{t=1}^{l_{n}} \mathbf{X}_{t}^{2} \mathbf{1}\left(\left\|\mathbf{X}_{t}\right\|>\varepsilon a_{n}\right)>\gamma a_{n}^{2}\right) \leq k_{n} l_{n} \mathbb{P}\left(\left\|\mathbf{X}_{t}\right\|>\varepsilon a_{n}\right) \leq c l_{n} / m_{n}=o(1) .
$$

Relation (A.1) turns into

$$
\mathbb{E} \mathrm{e}^{i \mathbf{s}^{\prime} a_{n}^{-2} \sum_{t=1}^{n} \mathbf{X}_{t}^{2} \mathbf{1}\left(\left\|\mathbf{X}_{t}\right\|>\varepsilon a_{n}\right)}-\left(\mathbb{E} \mathrm{e}^{i \mathbf{s}^{\prime} a_{n}^{-2} \sum_{t=1}^{m_{n}} \mathbf{X}_{t}^{2} \mathbf{1}\left(\left\|\mathbf{X}_{t}\right\|>\varepsilon a_{n}\right)}\right)^{k_{n}} \rightarrow 0, \quad \mathbf{s} \in \mathbb{R}^{p} .
$$

In view of (3.7), it is not difficult to see that we can replace the sum in the former characteristic function by the sum over the index set $J_{n}=\left\{1, \ldots, m_{n}-l_{n}, m_{n}+1, \ldots, 2 m_{n}-l_{n}, \ldots,\right\} \subset$ $\{1, \ldots, n\}$ and in the latter characteristic function by the sum over the index set $\left\{1, \ldots, m_{n}-l_{n}\right\}$. Without loss of generality, we may assume that $n / m_{n}$ is an integer. Thus, it remains to show that the following difference converges to zero for every $\mathbf{s} \in \mathbb{R}^{p}$ :

$$
\begin{aligned}
\left|\mathbb{E}\left[\mathrm{e}^{i \mathbf{s}^{\prime} a_{n}^{-2} \sum_{t \in J_{n}} \mathbf{X}_{t}^{2} \mathbf{1}\left(\left\|\mathbf{X}_{t}\right\|>\varepsilon a_{n}\right)}\right]-\left(\mathbb{E}\left[\mathrm{e}^{i \mathbf{s}^{\prime} a_{n}^{-2} \sum_{t=1}^{m_{n}-l_{n}} \mathbf{X}_{t}^{2} \mathbf{1}\left(\left\|\mathbf{X}_{t}\right\|>\varepsilon a_{n}\right)}\right]\right)^{k_{n}}\right| \\
=\mid \sum_{v=1}^{k_{n}} \mathbb{E}\left[\prod_{j=1}^{v-1} \mathrm{e}^{i \mathbf{s}^{\prime} a_{n}^{-2} \sum_{t=(j-1) m_{n}+1}^{j m_{n}-l_{n}} \mathbf{X}_{t}^{2} \mathbf{1}\left(\left\|\mathbf{X}_{t}\right\|>\varepsilon a_{n}\right)}\right. \\
\left.\quad \times\left(\mathrm{e}^{i \mathbf{s}^{\prime} a_{n}^{-2} \sum_{t=(v-1) m_{n}+1}^{v m_{n}-l_{n}} \mathbf{X}_{t}^{2} \mathbf{1}\left(\left\|\mathbf{X}_{t}\right\|>\varepsilon a_{n}\right)}-\mathbb{E}\left[\mathrm{e}^{i \mathbf{s}^{\prime} a_{n}^{-2} \sum_{t=(v-1) m_{n}+1}^{v m_{n}-l_{n}} \mathbf{X}_{t}^{2} \mathbf{1}\left(\left\|\mathbf{X}_{t}\right\|>\varepsilon a_{n}\right)}\right]\right)\right] \\
\quad \times \prod_{j=v+1}^{k_{n}} \mathbb{E}\left[\mathrm{e}^{i \mathbf{s}^{\prime} a_{n}^{-2} \sum_{t=(j-1) m_{n}+1}^{j m_{n}-l_{n}} \mathbf{X}_{t}^{2} \mathbf{1}\left(\left\|\mathbf{X}_{t}\right\|>\varepsilon a_{n}\right)}\right] .
\end{aligned}
$$

In view of a standard inequality for covariances of strongly mixing sequences of bounded random variables (see Doukhan [26], page 3) the right-hand side is bounded by $c k_{n} \alpha_{l_{n}}$ which converges 
to zero by construction. Here and in what follows, $c$ stands for any positive constant whose value is not of interest. Its value may change from line to line. This finishes the proof of the mixing condition.

(3) Next, we check the anti-clustering condition (A.2) for $\left(\mathbf{X}_{t}\right)$ with normalization $\left(a_{n}\right)$, implying the corresponding condition for $\left(\mathbf{X}_{t}^{2}\right)$ with normalization $\left(a_{n}^{2}\right)$. By similar methods as for part (1) of the proof, assuming that $\|\cdot\|$ is the max-norm, we have

$$
\begin{aligned}
& \mathbb{P}\left(\max _{t=l, \ldots, m_{n}}\left\|\mathbf{X}_{t}\right\|>\gamma a_{n} \mid\left\|\mathbf{X}_{0}\right\|>\gamma a_{n}\right) \\
& \quad \leq \sum_{t=l}^{m_{n}} \mathbb{P}\left(\left\|\mathbf{X}_{t}\right\|>\gamma a_{n} \mid\left\|\mathbf{X}_{0}\right\|>\gamma a_{n}\right) \\
& \quad \leq c \sum_{t=l}^{m_{n}} \sum_{i=1}^{p} \sum_{j=1}^{p} \frac{\mathbb{P}\left(\left|X_{i t}\right|>\gamma a_{n},\left|X_{j 0}\right|>\gamma a_{n}\right)}{\mathbb{P}\left(|Z|>\gamma a_{n}\right)} \\
& \quad \leq c \sum_{t=l}^{m_{n}} \sum_{i=1}^{p} \sum_{j=1}^{p} \frac{\mathbb{P}\left(\max \left(\sigma_{i t}, \sigma_{j 0}\right) \min \left(\left|Z_{i t}\right|,\left|Z_{j 0}\right|\right)>\gamma a_{n}\right)}{\mathbb{P}\left(|Z|>\gamma a_{n}\right)} \\
& \quad \leq c \sum_{t=l}^{m_{n}} \sum_{i=1}^{p} \sum_{j=1}^{p} \frac{\mathbb{P}\left(\sigma_{i t} \min \left(\left|Z_{i t}\right|,\left|Z_{j 0}\right|\right)>\gamma a_{n}\right)}{\mathbb{P}\left(|Z|>\gamma a_{n}\right)} .
\end{aligned}
$$

By stationarity, the probabilities on the right-hand side do not depend on $t \geq l$. Therefore and by Breiman's result, the right-hand side is bounded by

$$
c m_{n} \frac{\mathbb{P}\left(\min \left(\left|Z_{i t}\right|,\left|Z_{j 0}\right|\right)>\gamma a_{n}\right)}{\mathbb{P}\left(|Z|>\gamma a_{n}\right)}=O\left(\left(m_{n} / n\right)\left[n \mathbb{P}\left(|Z|>a_{n}\right)\right]\right)=o(1) .
$$

This proves (A.2) for $\left(\mathbf{X}_{t}\right)$.

(4) Next, we check the vanishing small values condition (A.3) for the partial sums of $\left(\mathbf{X}_{t}^{2}\right)$ and $\alpha \in(2,4)$. It is not difficult to see that it suffices to prove the corresponding result for the component processes:

$$
\begin{aligned}
& \underset{\varepsilon \downarrow 0}{\lim \limsup _{n \rightarrow \infty} \mathbb{P}}\left(\left|\sum_{t=1}^{n}\left(X_{i t}^{2} \mathbf{1}\left(\left|X_{i t}\right| \leq \varepsilon a_{n}\right)-\mathbb{E}\left[X_{i t}^{2} \mathbf{1}\left(\left|X_{i t}\right| \leq \varepsilon a_{n}\right)\right]\right)\right|>\gamma a_{n}^{2}\right)=0, \\
& \quad \gamma>0, i=1, \ldots, p .
\end{aligned}
$$

We have

$$
\begin{aligned}
& a_{n}^{-2} \sum_{t=1}^{n} \sigma_{i t}^{2} \mathbb{E}\left[Z_{i t}^{2} \mathbf{1}\left(\left|X_{i t}\right| \leq \varepsilon a_{n}\right) \mid \sigma_{i t}\right]-a_{n}^{-2} n \mathbb{E}\left[X_{i t}^{2} \mathbf{1}\left(\left|X_{i t}\right| \leq \varepsilon a_{n}\right)\right] \\
& \quad=a_{n}^{-2} \sum_{t=1}^{n}\left(\sigma_{i t}^{2}-\mathbb{E}\left[\sigma_{i t}^{2}\right]\right) \mathbb{E}\left[Z^{2}\right]
\end{aligned}
$$




$$
\begin{aligned}
& -a_{n}^{-2} \sum_{t=1}^{n}\left(\sigma_{i t}^{2} \mathbb{E}\left[Z_{i t}^{2} \mathbf{1}\left(\left|X_{i t}\right|>\varepsilon a_{n}\right) \mid \sigma_{i t}\right]-\mathbb{E}\left[X_{i t}^{2} \mathbf{1}\left(\left|X_{i t}\right|>\varepsilon a_{n}\right)\right]\right) \\
= & I_{1}+I_{2} .
\end{aligned}
$$

The sequence $\left(\sigma_{i t}^{2}\right)$ satisfies the central limit theorem with normalization $\sqrt{n}$. This follows from Ibragimov's central limit theorem for strongly mixing sequence whose rate function $\left(\alpha_{h}\right)$ satisfies (3.1) and has moment $\mathbb{E}\left[\sigma^{2(2+\delta))}\right]<\infty$ (see (3.2)); cf. Doukhan [26], page 45. We know that $\sqrt{n} / a_{n}^{2} \rightarrow 0$ for $\alpha \in(2,4)$. Therefore, $I_{1} \stackrel{\mathbb{P}}{\rightarrow} 0$. We also have

$$
\begin{aligned}
\mathbb{E}\left[I_{2}^{2}\right] \leq & \frac{n}{a_{n}^{4}} \mathbb{E}\left[\sigma^{4}\left(\mathbb{E}\left[Z^{2} \mathbf{1}\left(|X|>\varepsilon a_{n}\right) \mid \sigma\right]\right)^{2}\right] \\
& +2 \frac{n}{a_{n}^{4}} \sum_{h=1}^{n}\left|\operatorname{cov}\left(\sigma_{i 0}^{2} \mathbb{E}\left[Z_{i 0}^{2} \mathbf{1}\left(\left|X_{i 0}^{2}\right|>\varepsilon a_{n}\right) \mid \sigma_{i 0}\right], \sigma_{i h}^{2} \mathbb{E}\left[Z_{i h}^{2} \mathbf{1}\left(\left|X_{i h}^{2}\right|>\varepsilon a_{n}\right) \mid \sigma_{i h}\right]\right)\right| \\
= & I_{3}+I_{4} .
\end{aligned}
$$

In view of the moment conditions on $\sigma$ and since $\mathbb{E}\left[Z^{2}\right]<\infty, I_{3} \leq c\left(n / a_{n}^{4}\right) \rightarrow 0$. In view of Doukhan [26], Theorem 3 on page 9, we have

$$
I_{4} \leq c \frac{n}{a_{n}^{4}} \sum_{h=1}^{n} \alpha_{h}^{\delta /(2+\delta)}\left(\mathbb{E}|\sigma|^{2(2+\delta)}\right)^{2 /(2+\delta)} \rightarrow 0 .
$$

Thus, it suffices for (3.8) to prove

$$
\lim _{\varepsilon \downarrow 0} \limsup _{n \rightarrow \infty} \mathbb{P}\left(\left|\sum_{t=1}^{n}\left(\sigma_{i t}^{2} \mathbb{E}\left[Z_{i t}^{2} \mathbf{1}\left(\left|X_{i t}\right| \leq \varepsilon a_{n}\right) \mid \sigma_{i t}\right]-X_{i t}^{2} \mathbf{1}\left(\left|X_{i t}\right| \leq \varepsilon a_{n}\right)\right)\right|>\gamma a_{n}^{2}\right)=0,
$$

The summands are independent and centered, conditional on the $\sigma$-field generated by $\left(\sigma_{i t}\right)_{t=1, \ldots, n}$. An application of Čebyshev's inequality conditional on this $\sigma$-field and Karamata's theorem yield, as $n \rightarrow \infty$,

$$
\begin{aligned}
& \mathbb{E}\left[\mathbb{P}\left(\left|\sum_{t=1}^{n}\left(\sigma_{i t}^{2} \mathbb{E}\left[Z_{i t}^{2} \mathbf{1}\left(\left|X_{i t}\right| \leq \varepsilon a_{n}\right) \mid \sigma_{i t}\right]-X_{i t}^{2} \mathbf{1}\left(\left|X_{i t}\right| \leq \varepsilon a_{n}\right)\right)\right|>\gamma a_{n}^{2} \mid\left(\sigma_{i s}\right)\right)\right] \\
& \leq c a_{n}^{-4} \mathbb{E}\left[\sum_{t=1}^{n} \operatorname{var}\left(X_{i t}^{2} \mathbf{1}\left(\left|X_{i t}\right| \leq \varepsilon a_{n}\right) \mid \sigma_{i t}\right) \mid\left(\sigma_{i s}\right)\right] \\
& \leq c n \varepsilon^{4} \mathbb{E}\left[\left|X /\left(\varepsilon a_{n}\right)\right|^{4} \mathbf{1}\left(|X| \leq \varepsilon a_{n}\right)\right] \rightarrow c \varepsilon^{4-\alpha}
\end{aligned}
$$

The right-hand side converges to zero as $\varepsilon \downarrow 0$. 
This proves that all assumptions of Theorem A.1 are satisfied. Therefore, the random variables on the left-hand side of (3.3) converge to an $\alpha$-stable random vector with log-characteristic function

$$
\begin{aligned}
\int_{0}^{\infty} & \mathbb{E}\left[\mathrm{e}^{i y \mathbf{t}^{\prime} \sum_{j=0}^{\infty} \boldsymbol{\Theta}_{j}^{(2)}}-\mathrm{e}^{i y \mathbf{t}^{\prime} \sum_{j=1}^{\infty} \boldsymbol{\Theta}_{j}^{(2)}}-i y \mathbf{t}^{\prime} \mathbf{1}_{(1,2)}(\alpha / 2)\right] d\left(-y^{\alpha / 2}\right) \\
= & \sum_{j=1}^{p} \frac{1}{p} \int_{0}^{\infty} \mathbb{E}\left[\mathrm{e}^{i y t_{j}}-i y t_{j} \mathbf{1}_{(1,2)}(\alpha / 2)\right] d\left(-y^{\alpha / 2}\right), \quad \mathbf{t}=\left(t_{1}, \ldots, t_{p}\right)^{\prime} \in \mathbb{R}^{p},
\end{aligned}
$$

where we used (3.6) and that $\boldsymbol{\Theta}_{h}^{(2)}=\mathbf{0}$ for $h \geq 1$. One easily checks that all summands in this expression are homogeneous functions in $t_{j}$ of degree $\alpha / 2$. Therefore, the limiting random vector in (3.3) has the same distribution as the sum $\sum_{j=1}^{p} \mathbf{e}_{j} \xi_{j, \alpha / 2}$ for i.i.d. $\xi_{j, \alpha / 2}$ which are $\alpha / 2$-stable and totally skewed to the right (because all the summands in $S_{j}$ are non-negative).

\subsection{Eigenvalues of the sample covariance matrix}

We have the following approximations:

Theorem 3.6. Assume that one of the following conditions holds:

1. $\left(X_{i t}\right)$ is an i.i.d. field of regularly varying random variables with index $\alpha \in(0,4)$. If $\mathbb{E}[|X|]<\infty$ we also assume $\mathbb{E}[X]=0$.

2. $\left(X_{i t}\right)$ is a stochastic volatility model (2.1) satisfying the regular variation, mixing and moment conditions of Theorem 3.3. If $\mathbb{E}[|Z|]<\infty$ we also assume $\mathbb{E}[Z]=0$.

Then, with $\mathbf{X}^{n}$ as in (2.3),

$$
a_{n}^{-2}\left\|\mathbf{X}^{n}\left(\mathbf{X}^{n}\right)^{\prime}-\operatorname{diag}\left(\mathbf{X}^{n}\left(\mathbf{X}^{n}\right)^{\prime}\right)\right\|_{2} \stackrel{\mathbb{P}}{\rightarrow} 0
$$

where $\|\cdot\|_{2}$ is the spectral norm and $\left(a_{n}\right)$ is a sequence such that $n \mathbb{P}\left(|X|>a_{n}\right) \rightarrow 1$.

Proof. Part (1). Recall that for a $p \times p$ matrix $\mathbf{A}$ we have $\|\mathbf{A}\|_{2} \leq\|\mathbf{A}\|_{F}$, where $\|\cdot\|_{F}$ denotes the Frobenius norm. Hence,

$$
\begin{aligned}
a_{n}^{-4}\left\|\mathbf{X}^{n}\left(\mathbf{X}^{n}\right)^{\prime}-\operatorname{diag}\left(\mathbf{X}^{n}\left(\mathbf{X}^{n}\right)^{\prime}\right)\right\|_{2}^{2} & \leq a_{n}^{-4}\left\|\mathbf{X}^{n}\left(\mathbf{X}^{n}\right)^{\prime}-\operatorname{diag}\left(\mathbf{X}^{n}\left(\mathbf{X}^{n}\right)^{\prime}\right)\right\|_{F}^{2} \\
& =\sum_{1 \leq i \neq j \leq p}\left(a_{n}^{-2} S_{i j}\right)^{2} .
\end{aligned}
$$

In view of the assumptions, $\left(X_{i t} X_{j t}\right)_{t=1,2, \ldots}, i \neq j$, is an i.i.d. sequence of regularly varying random variables with index $\alpha$ which is also centered if $\mathbb{E}[|X|]<\infty$. We consider two different cases.

The case $\alpha \in(0,2)$. According to classical limit theory (see Feller [30], Petrov [40]) we have for $i \neq j, b_{n}^{-1} S_{i j} \stackrel{d}{\rightarrow} \xi_{\alpha}$, (see (2.3) for the definition of $S_{i j}$ ) where $\xi_{\alpha}$ is an $\alpha$-stable random variable and $\left(b_{n}\right)$ is chosen such that $n \mathbb{P}\left(\left|X_{1} X_{2}\right|>b_{n}\right) \rightarrow 1$ for independent copies $X_{1}, X_{2}$ of $X$. 
Since $\left(b_{n}\right)$ and $\left(a_{n}^{2}\right)$ are regularly varying with indices $1 / \alpha$ and $2 / \alpha$, respectively, the right-hand side in (3.9) converges to zero in probability.

The case $\alpha \in[2,4)$. In this case, the distribution of $X_{1} X_{2}$ is in the domain of attraction of the normal law. Since $X_{1} X_{2}$ has mean zero we can apply classical limit theory (see Feller [30], Petrov [40]) to conclude that $b_{n}^{-1} S_{i j} \stackrel{d}{\rightarrow} N$, where $\left(b_{n}\right)$ is regularly varying with index $1 / 2$ and $N$ is centered Gaussian. Since $b_{n} / a_{n}^{2} \rightarrow 0$ we again conclude that the right-hand side of (3.9) converges to zero in probability.

Part (2). We again appeal to (3.9). Let $\gamma<\min (2, \alpha)$. Then we have for $i \neq j$, using the independence of $\left(X_{i t} X_{j t}\right)$ conditional on $\left(\left(\sigma_{i t}, \sigma_{j t}\right)\right)$ and that the distribution of $Z$ is centered if its first absolute moments exists, that

$$
a_{n}^{-2 \gamma} \mathbb{E}\left[\left|S_{i j}\right|^{\gamma} \mid\left(\left(\sigma_{i t}, \sigma_{j t}\right)\right)\right] \leq c \frac{n}{a_{n}^{2 \gamma}} \frac{1}{n} \sum_{t=1}^{n}\left(\sigma_{i t} \sigma_{j t}\right)^{\gamma}\left(\mathbb{E}|Z|^{\gamma}\right)^{2},
$$

cf. von Bahr and Esséen [49] and Petrov [40], 2.6.20 on page 82. In view of the moment condition (3.2), we have $\mathbb{E}\left[\left(\sigma_{i} \sigma_{j}\right)^{\gamma}\right]<\infty$ and $n / a_{n}^{2 \gamma} \rightarrow 0$ if we choose $\gamma$ sufficiently close to $\min (2, \alpha)$. Then the right-hand side converges to zero in view of the ergodic theorem. An application of the conditional Markov inequality of order $\gamma$ yields $a_{n}^{-2} S_{i j} \stackrel{\mathbb{P}}{\rightarrow} 0$. This proves the theorem.

Corollary 3.7. Assume that $\left(X_{i t}\right)$ is either:

1. an i.i.d. field of regularly varying random variables with index $\alpha \in(0,4)$ and $\mathbb{E}[X]=0$ if $\mathbb{E}[|X|]<\infty$, or

2. a stochastic volatility model of regularly varying random variables with index $\alpha \in(0,4) \backslash$ \{2\} satisfying the conditions of Theorem 3.6(2).

Then

$$
a_{n}^{-2} \max _{i=1, \ldots, p}\left|\lambda_{(i)}-S_{(i)}\right| \stackrel{\mathbb{P}}{\rightarrow} 0
$$

where $\left(\lambda_{i}\right)$ are the eigenvalues of $\mathbf{X}^{n}\left(\mathbf{X}^{n}\right)^{\prime}, \lambda_{(1)} \geq \cdots \geq \lambda_{(p)}$ are their ordered values and $S_{(1)} \geq$ $\cdots \geq S_{(p)}$ are the ordered values of $S_{1}, \ldots, S_{p}$ defined in (2.3). In particular, we have

$$
a_{n}^{-2}\left(\lambda_{(1)}-c_{n}, \ldots, \lambda(p)-c_{n}\right) \stackrel{d}{\rightarrow}\left(\xi_{(1), \alpha / 2}, \ldots, \xi_{(p), \alpha / 2}\right),
$$

where $\left(c_{n}\right)$ is defined in (3.4) for $\alpha \neq 2$ and in Remark 3.4 for $\alpha=2,\left(\xi_{i, \alpha / 2}\right)$ are i.i.d. $\alpha / 2$-stable random variables given in Theorem 3.3 for the stochastic volatility model and in Remark 3.4 for the i.i.d. field, and $\xi_{(1), \alpha / 2} \geq \cdots \geq \xi_{(p), \alpha / 2}$ are their ordered values.

Proof. We have by Weyl's inequality (see Bhatia [9]) and Theorem 3.6,

$$
a_{n}^{-2} \max _{i=1, \ldots, p}\left|\lambda_{(i)}-S_{(i)}\right| \leq a_{n}^{-2}\left\|\mathbf{X}^{n}\left(\mathbf{X}^{n}\right)^{\prime}-\operatorname{diag}\left(\mathbf{X}^{n}\left(\mathbf{X}^{n}\right)^{\prime}\right)\right\|_{2} \stackrel{\mathbb{P}}{\rightarrow} 0
$$

If $\left(X_{i t}\right)$ is an i.i.d. random field (see Remark 3.4) or a stochastic volatility model satisfying the conditions of Theorem 3.6(2), we have (3.3). Then (3.11) implies (3.10). 
Remark 3.8. If $\alpha \in(2,4)$, we have $\mathbb{E}\left[X^{2}\right]<\infty$. Therefore, (3.10) reads as

$$
\frac{n}{a_{n}^{2}}\left(\frac{\lambda(i)}{n}-\mathbb{E}\left[X^{2}\right]\right)_{i=1, \ldots, p} \stackrel{d}{\rightarrow}\left(\xi_{(i), \alpha / 2}\right)_{i=1, \ldots, p} .
$$

We notice that $n / a_{n}^{2} \rightarrow \infty$ for $\alpha \in(2,4)$ since $\left(n / a_{n}^{2}\right)$ is regularly varying with index $1-2 / \alpha$. In particular, if $\operatorname{tr}\left(\mathbf{X}^{n}\left(\mathbf{X}^{n}\right)^{\prime}\right)$ denotes the trace of $\mathbf{X}^{n}\left(\mathbf{X}^{n}\right)^{\prime}$ we have for $i \leq p$,

$$
\frac{\lambda_{(i)}}{\operatorname{tr}\left(\mathbf{X}^{n}\left(\mathbf{X}^{n}\right)^{\prime}\right)}=\frac{\lambda_{(i)} / n}{\left(\lambda_{1}+\cdots+\lambda_{p}\right) / n} \stackrel{\mathbb{P}}{\rightarrow} \frac{1}{p}
$$

The joint asymptotic distribution of the ordered eigenvalues $\left(\lambda_{(i)}\right)$ is easily calculated from the distribution of a totally skewed $\alpha / 2$-stable random variable $\xi_{1, \alpha / 2}$; in particular, the limit of $\left(a_{n}^{-2}\left(\lambda_{(1)}-c_{n}\right)\right)$ has the distribution of $\max \left(\xi_{1, \alpha / 2}, \ldots, \xi_{p, \alpha / 2}\right)$.

For applications, it is more natural to replace the random variables $X_{i t}$ by their mean-centered versions $X_{i t}-\bar{X}_{i}$, where $\bar{X}_{i}=(1 / n) \sum_{t=1}^{n} X_{i t}$, instead of assuming that they have mean zero. The previous results remain valid for the sample-mean centered random variables $X_{i t}$, also in the case when $X$ has infinite first moment.

\subsection{Some applications: Limit results for ordered eigenvalues and eigenvectors of the sample covariance matrix}

In what follows, we assume the conditions of Corollary 3.7.

\subsubsection{Spacings}

Using the joint convergenceof the normalized ordered eigenvalues $\left(\lambda_{(i)}\right)$ we can calculate the limit of the spectral gaps:

$$
\left(\frac{\lambda_{(i)}-\lambda_{(i+1)}}{a_{n}^{2}}\right)_{i=1, \ldots, p-1} \stackrel{d}{\rightarrow}\left(\xi_{(i), \alpha / 2}-\xi_{(i+1), \alpha / 2}\right)_{i=1, \ldots, p-1} .
$$

We notice that the ordered values $\xi_{(i), \alpha / 2}$ and linear functionals thereof (such as $\xi_{(i), \alpha / 2}-$ $\left.\xi_{(i+1), \alpha / 2}\right)$ are again jointly regularly varying with index $\alpha / 2$. This is due to the continuous mapping theorem for regularly varying vectors; see Hult and Lindskog [32,33], cf. Jessen and Mikosch [35].

\subsubsection{Trace}

For the trace of $\mathbf{X}^{n}\left(\mathbf{X}^{n}\right)^{\prime}$ we have

$$
\begin{aligned}
a_{n}^{-2}\left(\operatorname{tr}\left(\mathbf{X}^{n}\left(\mathbf{X}^{n}\right)^{\prime}\right)-p c_{n}\right) & =a_{n}^{-2} \sum_{i=1}^{p}\left(S_{i}-c_{n}\right) \\
& =a_{n}^{-2} \sum_{i=1}^{p}\left(\lambda_{i}-c_{n}\right) \stackrel{d}{\rightarrow} \xi_{1, \alpha / 2}+\cdots+\xi_{p, \alpha / 2} \stackrel{d}{=} p^{2 / \alpha} \xi_{1, \alpha / 2} .
\end{aligned}
$$


Moreover, we have the joint convergenceof the normalized and centered $\left(\lambda_{(i)}\right)$ and $\operatorname{tr}\left(\mathbf{X}^{n}\left(\mathbf{X}^{n}\right)^{\prime}\right)=$ $\lambda_{1}+\cdots+\lambda_{p}$. In particular, we have the self-normalized limit relations

$$
\left(\frac{\lambda_{(i)}-c_{n}}{\operatorname{tr}\left(\mathbf{X}^{n}\left(\mathbf{X}^{n}\right)^{\prime}\right)-p c_{n}}\right)_{i=1, \ldots, p} \stackrel{d}{\rightarrow}\left(\frac{\xi_{(i), \alpha / 2}}{\xi_{1, \alpha / 2}+\cdots+\xi_{p, \alpha / 2}}\right)_{i=1, \ldots, p},
$$

and for $\alpha \in(2,4)$, by the strong law of large numbers,

$$
\frac{n p}{a_{n}^{2}}\left(\frac{\lambda_{(i)}-c_{n}}{\operatorname{tr}\left(\mathbf{X}^{n}\left(\mathbf{X}^{n}\right)^{\prime}\right)}\right)_{i=1, \ldots, p} \stackrel{d}{\rightarrow} \frac{\xi_{(i), \alpha / 2}}{\mathbb{E}\left[X^{2}\right]}
$$

\subsubsection{Determinant}

Since $\lambda_{i}-c_{n}$ are the eigenvalues of $\mathbf{X}^{n}\left(\mathbf{X}^{n}\right)^{\prime}-c_{n} \mathbf{I}_{p}$, where $\mathbf{I}_{p}$ is the $p \times p$ identity matrix, we obtain for the determinant

$$
\begin{aligned}
\operatorname{det}\left(a_{n}^{-2}\left(\mathbf{X}^{n}\left(\mathbf{X}^{n}\right)^{\prime}-c_{n} \mathbf{I}_{p}\right)\right) & =\prod_{i=1}^{p} a_{n}^{-2}\left(\lambda_{(i)}-c_{n}\right) \\
& \stackrel{d}{\rightarrow} \xi_{(1), \alpha / 2} \cdots \xi_{(p), \alpha / 2}=\xi_{1, \alpha / 2} \cdots \xi_{p, \alpha / 2} .
\end{aligned}
$$

For $\alpha \in(2,4)$, we also have

$$
\begin{aligned}
\frac{1}{a_{n}^{2} c_{n}^{p-1}}\left(\operatorname{det}\left(\mathbf{X}^{n}\left(\mathbf{X}^{n}\right)^{\prime}\right)-c_{n}^{p}\right) & =\sum_{i=1}^{p} a_{n}^{-2}\left(\lambda_{(i)}-c_{n}\right) \prod_{j=1}^{i-1} \frac{\lambda_{(j)}}{c_{n}} \\
& \stackrel{d}{\rightarrow} \sum_{i=1}^{p} \xi_{(i), \alpha / 2}=\sum_{i=1}^{p} \xi_{i, \alpha / 2} \stackrel{d}{=} p^{2 / \alpha} \xi_{1, \alpha / 2},
\end{aligned}
$$

where we used (3.12).

\subsubsection{Eigenvectors}

It is also possible to localize the eigenvectors of the matrix $a_{n}^{-2} \mathbf{X}^{n}\left(\mathbf{X}^{n}\right)^{\prime}$. Since this matrix is approximated by its diagonal in spectral norm, one may expect that the unit eigenvectors of the original matrix are close to the canonical basis vectors. We can write

$$
a_{n}^{-2} \mathbf{X}^{n}\left(\mathbf{X}^{n}\right)^{\prime} \mathbf{e}_{L_{j}}=a_{n}^{-2} S_{(j)} \mathbf{e}_{L_{j}}+\varepsilon_{n} \mathbf{W}
$$

where $\mathbf{W}$ is a unit vector orthogonal to $\mathbf{e}_{L_{j}}, L_{j}$ is the index of $S_{(j)}=S_{L_{j}}$ and

$$
\varepsilon_{n}=a_{n}^{-2}\left\|\left(\mathbf{X}^{n}\left(\mathbf{X}^{n}\right)^{\prime}-S_{(j)}\right) \mathbf{e}_{L_{j}}\right\|_{\ell_{2}} \stackrel{\mathbb{P}}{\rightarrow} 0
$$


from Theorem 3.6 and by equivalence of all matrix norms. According to Proposition A.1 in Benaych-Georges and Peché [7], there is an eigenvalue $a_{n}^{-2} \lambda_{(j)}$ of $a_{n}^{-2} \mathbf{X}^{n}\left(\mathbf{X}^{n}\right)^{\prime}$ in some $\varepsilon_{n}$ neighborhood of $a_{n}^{-2} S_{(j)}$. Define

$$
\Omega_{n}=\left\{a_{n}^{-2}\left|\lambda_{(j)}-\lambda_{(l)}\right|>d_{n}, l \neq j\right\}
$$

for $d_{n}=k \varepsilon_{n}$ for any fixed $k>1$. Then $\lim _{n \rightarrow \infty} \mathbb{P}\left(\Omega_{n}\right)=1$ because of (3.14) and $d_{n} \stackrel{\mathbb{P}}{\rightarrow} 0$. Hence, for large $n, a_{n}^{-2} \lambda_{(j)}$ and $a_{n}^{-2} \lambda_{(l)}$ have distance at least $d_{n}$ with high probability. Another application of Proposition A.1 in [7] yields that the unit eigenvector $\mathbf{V}$ associated with $a_{n}^{-2} \lambda_{(j)}$ satisfies the relation

$$
\begin{aligned}
\limsup _{n \rightarrow \infty} \mathbb{P}\left(\left\|\mathbf{V}-V_{L_{j}} \mathbf{e}_{L_{j}}\right\|_{\ell_{2}}>\delta\right) & \leq \limsup _{n \rightarrow \infty} \mathbb{P}\left(\left\{\left\|\mathbf{V}-V_{L_{j}} \mathbf{e}_{L_{j}}\right\|_{\ell_{2}}>\delta\right\} \cap \Omega_{n}\right)+\limsup _{n \rightarrow \infty} \mathbb{P}\left(\Omega_{n}^{c}\right) \\
& \leq \limsup _{n \rightarrow \infty} \mathbb{P}\left(\left\{2 \varepsilon_{n} /\left(d_{n}-\varepsilon_{n}\right)>\delta\right\} \cap \Omega_{n}\right) \\
& =\mathbf{1}_{\{2 /(k-1)>\delta\} .}
\end{aligned}
$$

For any fixed $\delta>0$, the right-hand side is zero for sufficiently large $k$. Since both $\mathbf{V}$ and $\mathbf{e}_{L_{j}}$ are unit eigenvectors this means that $\left\|\mathbf{V}-\mathbf{e}_{L_{j}}\right\|_{\ell_{2}} \stackrel{\mathbb{P}}{\rightarrow} 0$.

\subsubsection{Sample correlation matrix}

In Remark 3.8, we mentioned that we can replace the variables $X_{i t}$ by their sample-mean centered versions $X_{i t}-\bar{X}_{i}$ without changing the asymptotic theory. Similarly, one may be interested in transforming the $X_{i t}$ as follows:

$$
\tilde{X}_{i t}=\frac{X_{i t}-\bar{X}_{i}}{\widehat{\sigma}_{i}}, \quad \widehat{\sigma}_{i}^{2}=\sum_{t=1}^{n}\left(X_{i t}-\bar{X}_{i}\right)^{2} .
$$

Then the matrix

$$
\widetilde{\mathbf{X}}^{n}\left(\widetilde{\mathbf{X}}^{n}\right)^{\prime}=\left(\sum_{t=1}^{n} \widetilde{X}_{i t} \widetilde{X}_{j t}\right)_{i, j=1, \ldots, p},
$$

is the sample correlation matrix. We write $\tilde{\lambda}_{i}, i=1, \ldots, p$, for the eigenvalues of $\widetilde{\mathbf{X}}^{n}\left(\widetilde{\mathbf{X}}^{n}\right)^{\prime}$ and $\widetilde{\lambda}_{(1)} \geq \cdots \geq \widetilde{\lambda}_{(p)}$ for their ordered values.

We notice that the entries of this matrix are all bounded in modulus by one. In particular, the diagonal consists of ones. We do not have a complete limit theory for the eigenvalues $\tilde{\lambda}_{i}$. We restrict ourselves to i.i.d. $\left(X_{i t}\right)$ to explain the differences.

Lemma 3.9. Assume that $\left(X_{i t}\right)$ is an i.i.d. field of random variables.

1. If $\mathbb{E}\left[X^{2}\right]<\infty$, then

$$
\sqrt{n} \max _{i=1, \ldots, p}\left|\tilde{\lambda}_{i}-1\right|=O_{\mathbb{P}}(1)
$$


2. If $X$ is regularly varying with index $\alpha \in(0,2)$, then

$$
\frac{a_{n}^{2}}{b_{n}} \max _{i=1, \ldots, p}\left|\tilde{\lambda}_{i}-1\right|=O_{\mathbb{P}}(1),
$$

where $\left(a_{n}\right)$ and $\left(b_{n}\right)$ are chosen such that $\mathbb{P}\left(|X|>a_{n}\right) \sim \mathbb{P}\left(\left|X_{1} X_{2}\right|>b_{n}\right) \sim n^{-1}$ for i.i.d. copies $X_{1}, X_{2}$ of $X$.

Remark 3.10. Notice that the lemma implies $\tilde{\lambda}_{i} \stackrel{\mathbb{P}}{\rightarrow} 1$ for $i=1, \ldots, p$, and the analog of relation (3.13) remains valid.

Proof. Part (1) We assume without loss of generality that $1=\mathbb{E}\left[X^{2}\right]$. Then by classical limit theory,

$$
\begin{aligned}
\sqrt{n}\left(\widetilde{\mathbf{X}}^{n}\left(\widetilde{\mathbf{X}}^{n}\right)^{\prime}-\operatorname{diag}\left(\widetilde{\mathbf{X}}^{n}\left(\widetilde{\mathbf{X}}^{n}\right)^{\prime}\right)\right) & =\sqrt{n}\left(\widetilde{\mathbf{X}}^{n}\left(\widetilde{\mathbf{X}}^{n}\right)^{\prime}-\mathbf{I}_{p}\right) \\
& =\left(\mathbf{1}(i \neq j) \frac{n^{-1 / 2} \sum_{t=1}^{n}\left(X_{i t}-\bar{X}_{i}\right)\left(X_{j t}-\bar{X}_{j}\right)}{\left(\widehat{\sigma}_{i} / \sqrt{n}\right)\left(\widehat{\sigma}_{j} / \sqrt{n}\right)}\right) \\
& \stackrel{d}{\rightarrow}\left(N_{i j} \mathbf{1}(i \neq j)\right),
\end{aligned}
$$

where $N_{i j}, 1 \leq i<j \leq n$, are i.i.d. $N(0,1)$ and $N_{i j}=N_{j i}$. By Weyl's inequality,

$$
\sqrt{n} \max _{i=1, \ldots, p}\left|\widetilde{\lambda}_{(i)}-1\right| \leq \sqrt{n}\left\|\widetilde{\mathbf{X}}^{n}\left(\widetilde{\mathbf{X}}^{n}\right)^{\prime}-\mathbf{I}_{p}\right\|_{2}=O_{\mathbb{P}}(1) .
$$

Part (2) If $X$ is regularly varying with index $\alpha \in(0,2)$, we have that $\left(a_{n}^{-2} \widehat{\sigma}_{i}^{2}\right)$ converges to a vector of i.i.d. positive $\alpha / 2$-stable random variables $\left(\xi_{i}\right)$, while for every $i \neq j, b_{n}^{-1} \sum_{t=1}^{n}\left(X_{i t}-\right.$ $\left.\bar{X}_{i}\right)\left(X_{j t}-\bar{X}_{j}\right) \stackrel{d}{\rightarrow} \xi_{i j}$ and the limit $\xi_{i j}$ is $\alpha$-stable. Then by Weyl's inequality

$$
\frac{a_{n}^{2}}{b_{n}} \max _{i=1, \ldots, p}\left|\tilde{\lambda}_{(i)}-1\right| \leq \frac{a_{n}^{2}}{b_{n}}\left\|\widetilde{\mathbf{X}}^{n}\left(\widetilde{\mathbf{X}}^{n}\right)^{\prime}-\mathbf{I}_{p}\right\|_{2}=O_{\mathbb{P}}(1) .
$$

\section{Case (2): $\sigma$ dominates the tail}

In this section, we assume the conditions of Case (2); see Section 2.2. Our goal is to derive results analogous to Case (1): regular variation of $\left(X_{i t}\right)$, infinite variance limits for $S_{i j}$ and limit theory for the eigenvalues of the corresponding sample covariance matrices. It turns out that this case offers a wider spectrum of possible limit behaviors and that we have to further distinguish our assumptions about the distribution of the innovations $\left(\eta_{i}\right)$ with generic element $\eta$ in the log-linear model for $\sigma$; see (2.7). So, in addition to (2.8) we assume that either

$$
\mathbb{E}\left[\mathrm{e}^{\eta \alpha}\right]=\infty
$$


or

$$
\lim _{x \rightarrow \infty} \frac{\mathbb{P}\left(\eta_{1}+\eta_{2}>x\right)}{\mathbb{P}\left(\eta_{1}>x\right)}=c \in(0, \infty) \quad \Leftrightarrow \quad \lim _{x \rightarrow \infty} \frac{\mathbb{P}\left(\mathrm{e}^{\eta_{1}} \cdot \mathrm{e}^{\eta_{2}}>x\right)}{\mathbb{P}\left(\mathrm{e}^{\eta_{1}}>x\right)}=c \in(0, \infty)
$$

hold, where $\eta_{1}$ and $\eta_{2}$ are independent copies of $\eta$.

Remark 4.1. Following Cline [14], we call the distribution of a random variable $\eta$ convolution equivalent if $\mathrm{e}^{\eta}$ is regularly varying and relation (4.2) holds. The assumptions (4.1) and (4.2) are mutually exclusive, since the only possible finite limit $c$ in (4.2) is given by $c=2 \mathbb{E}\left[\mathrm{e}^{\eta \alpha}\right]$; see Davis and Resnick [16]. There are, however, regularly varying distributions of $\mathrm{e}^{\eta}$ which satisfy $\mathbb{E}\left[\mathrm{e}^{\eta \alpha}\right]<\infty$ but not (4.2). An example is given in Cline [14], page 538; see also Lemma B.1(3) for a necessary and sufficient condition ensuring (4.2).

As we will see later, relations (4.1) and (4.2) cause rather distinct limit behavior of the sample covariance matrix. In particular, (4.2) allows for non-vanishing off-diagonal elements of the normalized sample covariance matrices, in contrast to Case (1).

For notational simplicity, define

$$
\psi=\max _{k, l} \psi_{k l} \quad \text { and } \quad \Lambda=\left\{(k, l): \psi_{k l}=\psi\right\}
$$

Recall that for convenience we assume that $\psi=1$; if the latter condition does not hold we can replace (without loss of generality) the random variables $\eta_{k l}$ by $\psi \eta_{k l}$ and the coefficients $\psi_{k l}$ by $\psi_{k l} / \psi$. For given $(i, j)$, we define

$$
\psi^{i j}=\max _{k, l}\left(\psi_{k l}+\psi_{k+i-j, l}\right)
$$

Notice that $1 \leq \psi^{i j} \leq 2$. For $d \geq 1$, we write $\mathbf{i}=\left(i_{1}, \ldots, i_{d}\right), \mathbf{j}=\left(j_{1}, \ldots, j_{d}\right)$ for elements of $\mathbb{Z}^{d}$. For given $\mathbf{i}$ and $\mathbf{j}$ we also define

$$
\psi^{\mathbf{i}, \mathbf{j}}=\max _{1 \leq l \leq d} \psi^{i_{l}, j_{l}}
$$

\subsection{Regular variation}

We start by showing that the volatility sequences are regularly varying.

Proposition 4.2. Under the aforementioned conditions and conventions (including that either (4.1) or (4.2) hold):

1. each of the sequences $\left(\sigma_{i t}\right)_{t \in \mathbb{Z}}, i=1,2, \ldots$, is regularly varying with index $\alpha$,

2. each of the sequences $\left(\sigma_{i t} \sigma_{j t}\right)_{t \in \mathbb{Z}}, i, j=1,2, \ldots$, is regularly varying with corresponding index $\alpha / \psi^{i j}$,

3. for $d \geq 1$ and $\mathbf{i}, \mathbf{j} \in \mathbb{Z}$, the $d$-variate sequence $\left(\left(\sigma_{i_{k}, t} \sigma_{j_{k}, t}\right)_{1 \leq k \leq d}\right)_{t \in \mathbb{Z}}$ is regularly varying with index $\alpha / \psi^{\mathbf{i}, \mathbf{j}}$. 
Remark 4.3. Part (3) of the proposition possibly includes degenerate cases in the sense that for some choices of $\left(i_{k}, j_{k}\right),\left(\sigma_{i_{k}, t} \sigma_{j_{k}, t}\right)$ is regularly varying with index $\alpha / \psi^{i_{k}, j_{k}}>\alpha / \psi^{\mathbf{i}, \mathbf{j}}$.

Part (3) implies (2) in the case $d=1$. Part (2) implies (1) by setting $i=j$ and observing that, by non-negativity of $\sigma$, regular variation of $\left(\sigma_{i t}^{2}\right)$ with index $\alpha / 2$ is equivalent to regular variation of $\left(\sigma_{i t}\right)$ with index $\alpha$.

Proof. To give some intuition we start with the proof of the marginal regular variation of $\sigma$, although it is just a special case of (1). We have

$$
\sigma_{i t}=\mathrm{e}^{\sum_{(k, l) \in \Lambda} \eta_{i-k, t-l}} \mathrm{e}^{\sum_{(k, l) \notin \Lambda} \psi_{k l} \eta_{i-k, t-l}}=: \sigma_{i t, \Lambda} \sigma_{i t, \Lambda^{c}} .
$$

We first verify that $\sigma=\sigma_{\Lambda} \sigma_{\Lambda^{c}}$ is regularly varying with index $\alpha$. Since $|\Lambda|<\infty$ by our assumptions, and in view of Embrechts and Goldie [28], Corollary on page 245, cf. also Lemma B.1(1) below, the product $\sigma_{\Lambda}$ is regularly varying with index $\alpha$. The random variable $\sigma_{\Lambda^{c}}$ is independent of $\sigma_{\Lambda}$. Similarly to Mikosch and Rezapour [38] (see also the end of this proof for a similar argumentation) one can show that $\sigma_{\Lambda^{c}}$ has moment of order $\alpha+\varepsilon$ for sufficiently small positive $\varepsilon$. Therefore, by Breiman's lemma [11],

$$
\mathbb{P}(\sigma>x) \sim \mathbb{E}\left[\sigma_{\Lambda^{c}}^{\alpha}\right] \mathbb{P}\left(\sigma_{\Lambda}>x\right), \quad x \rightarrow \infty
$$

This proves regular variation with index $\alpha$ of the marginal distributions of $\left(\sigma_{i t}\right)$.

In the remainder of the proof, we focus on (3). For a given choice of $\mathbf{i}, \mathbf{j}, \mathbf{t} \in \mathbb{Z}^{d}$, we write

$$
\Lambda_{\mathbf{i}, \mathbf{j}, \mathbf{t}}=\left\{(m, n): \psi_{i_{l}-m, t_{l}-n}+\psi_{j_{l}-m, t_{l}-n}=\psi^{\mathbf{i}, \mathbf{j}} \text { for some } 1 \leq l \leq d\right\} .
$$

We will show that the random vector $\left(\sigma_{i_{1}, t_{1}} \sigma_{j_{1}, t_{1}}, \ldots, \sigma_{i_{d}, t_{d}} \sigma_{j_{d}, t_{d}}\right)=: \sigma^{\prime}$ is regularly varying with index $\alpha / \psi^{\mathbf{i}, \mathbf{j}}$ which proves (3). Note that

$$
\begin{aligned}
\sigma_{i, t} \sigma_{j, t} & =\prod_{(k, l)} \exp \left(\psi_{k l} \eta_{i-k, t-l}\right) \prod_{\left(k^{\prime}, l^{\prime}\right)} \exp \left(\psi_{k^{\prime} l^{\prime}} \eta_{j-k^{\prime}, t-l^{\prime}}\right) \\
& =\prod_{(m, n)} \exp \left(\left(\psi_{i-m, t-n}+\psi_{j-m, t-n}\right) \eta_{m, n}\right)
\end{aligned}
$$

and write

$$
\boldsymbol{\sigma}=\operatorname{diag}\left(\left(\begin{array}{cc}
\prod_{(m, n) \in \Lambda_{\mathbf{i}, \mathbf{j}, \mathbf{t}}^{c}} \mathrm{e}^{\eta_{m, n}\left(\psi_{i_{1}-m, t_{1}-n}+\psi_{j_{1}-m, t_{1}-n}\right)} \\
\vdots \\
\prod_{(m, n) \in \Lambda_{\mathbf{i}, \mathbf{j}, \mathbf{t}}^{c}} \mathrm{e}^{\eta_{m, n}\left(\psi_{i_{d}-m, t_{d}-n}+\psi_{j_{d}-m, t_{d}-n}\right)}
\end{array}\right)\right)
$$




$$
\times\left(\begin{array}{cc}
\prod_{(m, n) \in \Lambda_{\mathbf{i}, \mathbf{j}, \mathbf{t}}} \mathrm{e}^{\eta_{m, n}\left(\psi_{i_{1}-m, t_{1}-n}+\psi_{j_{1}-m, t_{1}-n}\right)} \\
\prod_{(m, n) \in \Lambda_{\mathbf{i}, \mathbf{j}, \mathbf{t}}} \mathrm{e}^{\eta_{m, n}\left(\psi_{i_{d}-m, t_{d}-n}+\psi_{j_{d}-m, t_{d}-n}\right)}
\end{array}\right)
$$

$$
=: \mathbf{A Z} \text {, }
$$

where $\operatorname{diag}\left(\left(a_{1}, \ldots, a_{k}\right)\right)$ is any diagonal matrix with diagonal elements $a_{1}, \ldots, a_{k}$. We notice that $\mathbf{A}$ and $\mathbf{Z}$ are independent.

Consider i.i.d. copies $\left(Y_{j}\right)$ of $\mathrm{e}^{\eta}$. There exist suitable numbers $\left(a_{i j}\right)_{1 \leq i \leq d, 1 \leq j \leq p}$ with $p=$ $\left|\Lambda_{\mathbf{i}, \mathbf{j}, \mathbf{t}}\right|$ such that the components of $\mathbf{Z}$ have representation in distribution $\prod_{j=1}^{p} Y_{j}^{a_{i j}}, 1 \leq i \leq d$. By assumption, $Y_{j}$ is regularly varying with index $\alpha$ and satisfies either assumption (B.3) or $\mathbb{E}\left[Y_{j}^{\alpha}\right]=\infty$. Furthermore, for each $j$ there exists one $1 \leq i \leq d$ such that $a_{i j}=a_{\max }=\psi^{\mathbf{i}, \mathbf{j}}$ by the definition of $\Lambda_{\mathbf{i}, \mathbf{j}, \mathbf{t}}$. An application of Proposition B.3 shows that $\mathbf{Z}$ is regularly varying with index $\alpha / \psi^{\mathbf{i}, \mathbf{j}}$ and limit measure $\mu_{\mathbf{Z}}$ which is given as $\mu$ in Proposition B.3(ii) (if (4.1) holds) or Proposition B.3(i) (if (4.2) holds). Now, choose $\epsilon, \delta>0$ such that

$$
\frac{\psi_{i_{l}-m, t_{l}-n}+\psi_{j_{l}-m, t_{l}-n}}{\psi^{\mathbf{i}, \mathbf{j}}}(1+\delta)<1-\epsilon, \quad(m, n) \in \Lambda_{\mathbf{i}, \mathbf{j}, \mathbf{t}}^{c}, 1 \leq l \leq d,
$$

which is possible by the definition of $\Lambda_{\mathbf{i}, \mathbf{j}, \mathbf{t}}$ and the summability constraint on the coefficients. Then we have

$$
\begin{aligned}
\mathbb{E} & {\left[\|\mathbf{A}\|_{\mathrm{op}}^{\left.\alpha(1+\delta) / \psi^{\mathbf{i}, \mathbf{j}}\right]}\right.} \\
& \leq \sum_{l=1}^{d} \prod_{(m, n) \in \Lambda_{\mathbf{i}, \mathbf{j}, \mathbf{t}}^{c}} \mathbb{E}\left[\mathrm{e}^{\eta_{m, n} \alpha(1+\delta)\left(\psi_{i_{l}-m, t_{l}-n}+\psi_{j_{l}-m, t_{l}-n}\right) / \psi^{\mathbf{i}, \mathbf{j}}}\right] \\
& \leq \sum_{l=1}^{d} \prod_{(m, n) \in \Lambda_{\mathbf{i}, \mathbf{j}, \mathbf{t}}^{c}} \mathbb{E}\left[\mathrm{e}^{\eta_{m, n} \alpha(1-\epsilon)}\right]^{(1+\delta)\left(\psi_{i_{l}-m, t_{l}-n}+\psi_{j_{l}-m, t_{l}-n}\right) /\left((1-\epsilon) \psi^{\mathbf{i}, \mathbf{j}}\right)}<\infty
\end{aligned}
$$

where we used Jensen's inequality for the penultimate step and the summability condition of the coefficients for the final one. Thus, we have verified all conditions of the multivariate Breiman lemma in Basrak et al. [4], implying that $\sigma$ inherits regular variation from $\mathbf{Z}$ with corresponding index $\alpha / \psi^{\mathbf{i}, \mathbf{j}}$ and limit measure $\mu_{\boldsymbol{\sigma}}(\cdot)=\mathbb{E}\left[\mu_{\mathbf{Z}}\left(\mathbf{A}^{-1} \cdot\right)\right]$.

Proposition 4.4. Assume that the aforementioned conditions (including either (4.1) or (4.2)) hold and that in addition $\mathbb{E}\left[|Z|^{\alpha+\delta}\right]<\infty$ for some $\delta>0$. Then the following statements hold:

1. Each of the sequences $\left(X_{i t}\right)_{t \in \mathbb{Z}}, i \in \mathbb{Z}$, is regularly varying with index $\alpha$.

If (4.1) holds, then the corresponding spectral tail process satisfies $\Theta_{t}^{i}=0$ a.s., $t \geq 1$, and $\mathbb{P}\left(\Theta_{0}^{i}= \pm 1\right)=\mathbb{E}\left[Z_{ \pm}^{\alpha}\right] / \mathbb{E}\left[|Z|^{\alpha}\right]$. 
If (4.2) holds, then for any Borel set $B=B_{0} \times \cdots \times B_{n} \subset \mathbb{R}^{n+1}$,

$$
\begin{aligned}
& \mathbb{P}\left(\left(\Theta_{t}^{i}\right)_{t=0, \ldots, n} \in B\right) \\
& \quad=\sum_{(u, v) \in \Lambda_{i}^{(0)}} \frac{1}{\left|\Lambda_{i}^{(0)}\right|} \frac{\mathbb{E}\left[\mathbf{1}\left(\left(\mathbf{1}\left((u, v) \in \Lambda_{i}^{(t)}\right) \frac{X_{i t}}{\left|X_{i 0}\right|}\right)_{t=0, \ldots, n} \in B\right)\left|X_{i 0}\right|^{\alpha}\right]}{\mathbb{E}\left[\left|X_{i 0}\right|^{\alpha}\right]},
\end{aligned}
$$

where $\Lambda_{i}^{(t)}=\left\{(u, v): \psi_{i-u, t-v}=1\right\}, t=0, \ldots, n$.

2. Each of the sequences $\left(X_{i t} X_{j t}\right)_{t \in \mathbb{Z}}, i, j \in \mathbb{Z}$, is regularly varying with index $\alpha / \psi^{i j}$.

If (4.1) holds, then the corresponding spectral tail process satisfies $\Theta_{t}^{i j}=0$ a.s., $t \geq 1$, and $\mathbb{P}\left(\Theta_{0}^{i j}= \pm 1\right)=\mathbb{E}\left[\left(Z_{i} Z_{j}\right)_{ \pm}^{\alpha / \psi^{i j}}\right] / \mathbb{E}\left[\left|Z_{i} Z_{j}\right|^{\alpha / \psi^{i j}}\right]$.

If (4.2) holds, then for any Borel set $B=B_{0} \times \cdots \times B_{n} \subset \mathbb{R}^{n+1}$,

$$
\begin{aligned}
& \mathbb{P}\left(\left(\Theta_{t}^{i j}\right)_{t=0, \ldots, n} \in B\right) \\
& \quad=\sum_{(u, v) \in \Lambda_{i, j}^{(0)}} \frac{1}{\left|\Lambda_{i, j}^{(0)}\right|} \frac{\mathbb{E}\left[\mathbf{1}\left(\left(\mathbf{1}\left((u, v) \in \Lambda_{i, j}^{(t)}\right) \frac{X_{i t} X_{j t}}{\left|X_{i 0} X_{j 0}\right|}\right)_{t=0, \ldots, n} \in B\right)\left|X_{i 0} X_{j 0}\right|^{\alpha / \psi^{i j}}\right]}{\mathbb{E}\left[\left|X_{i 0} X_{j 0}\right|^{\left.\alpha / \psi^{i j}\right]}\right.},
\end{aligned}
$$

where $\Lambda_{i, j}^{(t)}=\left\{(u, v): \psi_{i-u, t-v}+\psi_{j-u, t-v}=\psi^{i j}\right\}, t=0, \ldots, n$.

3. For $d \geq 1$ and $\mathbf{i}, \mathbf{j} \in \mathbb{Z}^{d}$, the $d$-variate sequence $\left(\left(X_{i_{k} t} X_{j_{k} t}\right)_{1 \leq k \leq d}\right)_{t \in \mathbb{Z}}$ is jointly regularly varying with index $\alpha / \psi^{\mathbf{i}, \mathbf{j}}$.

Remark 4.5. 1. Equation (4.7) shows that in this case the distribution of $\left(\Theta_{t}^{i}\right)_{t \geq 0}$ is a mixture of $\left|\Lambda_{i}^{(0)}\right|$ distributions, where each distribution gets the weight $1 /\left|\Lambda_{i}^{(0)}\right|$. Heuristically speaking, a distribution in this mixture that corresponds to a specific $(u, v) \in \Lambda_{i}^{(0)}$ has interpretation as the distribution of $\left(X_{i t} /\left|X_{i 0}\right|\right)_{t \geq 0}$, given that we have seen an extreme observation of $\left|X_{i 0}\right|$ caused by an extreme realization of $\mathrm{e}^{\eta_{u, v}}$. The variables $\mathrm{e}^{\eta_{u, v}},(u, v) \in \Lambda_{i}^{(0)}$, are those which have a maximum exponent (equal to 1 ) in the product $\prod_{(u, v)} \exp \left(\psi_{i-u,-v} \eta_{u, v}\right)=\sigma_{i 0}$. They are therefore the factors which are most likely to make $\sigma_{i 0}$, hence $X_{i 0}$, extreme.

An analogous interpretation can be derived from (4.8) for the distribution of $\left(\Theta_{t}^{i j}\right)_{t \geq 0}$.

2. Note that for fixed $i, j$, the inner indicator functions in (4.7) and (4.8) are positive only for finitely many $t$. Hence, there are only finitely many $t \geq 1$ such that $\mathbb{P}\left(\Theta_{t}^{i} \neq 0\right)>0$ and $\mathbb{P}\left(\Theta_{t}^{(i j)} \neq 0\right)>0$.

3. Using similar techniques as in the proof of cases (1) and (2) below, one can also give an explicit expression for the resulting $d$-dimensional spectral tail process of $\left(\left(X_{i_{k} t} X_{j_{k} t}\right)_{1 \leq k \leq d}\right)_{t \in \mathbb{Z}}$ in (3). However, due to its complexity, we refrain from stating it here.

Proof. We start by showing that all mentioned sequences are regularly varying. Exemplarily, we show this for case (2). Very similar arguments can be used for the two other cases. For $n \geq 0$ write

$$
\left(X_{i t} X_{j t}\right)_{t=0, \ldots, n}^{\prime}=\operatorname{diag}\left(\left(Z_{i t} Z_{j t}\right)_{t=0, \ldots, n}^{\prime}\right) \cdot\left(\sigma_{i t} \sigma_{j t}\right)_{t=0, \ldots, n}^{\prime}
$$


Since $\psi^{i j} \geq 1$ our moment assumption on $Z$ implies that $\mathbb{E}\left[|Z|^{\alpha / \psi^{i j}+\delta}\right]<\infty$ for some $\delta>0$. Then Proposition 4.2 allows us to apply the aforementioned multivariate Breiman lemma, yielding the regular variation of the vector $\left(X_{i t} X_{j t}\right)_{t=0, \ldots, n}$ with index $\alpha / \psi^{i j}$. From the first definition given in Section 2.3, this implies the regular variation of the sequence.

As for the derivation of the explicit form of the spectral tail process in (1) and (2), we restrict ourselves to derive the distribution of the spectral tail process $\left(\Theta_{t}^{i j}\right)_{t \geq 0}$ in part (2); part (1) is similar.

If $\mu_{n}^{\sigma^{i j}}$ denotes the vague limit measure of $\left(\sigma_{i, 0} \sigma_{j, 0}, \ldots, \sigma_{i, n} \sigma_{j, n}\right)^{\prime}$ the multivariate Breiman lemma yields the vague limit measure $\mu_{n}^{\mathbf{X}^{i j}}$ of $\left(X_{i, 0} X_{j, 0}, \ldots, X_{i, n} X_{j, n}\right)^{\prime}$ given by

$$
\begin{aligned}
\mu_{n}^{\mathbf{X}^{i j}}(B) & =c \mathbb{E}\left[\mu_{n}^{\sigma^{i j}}\left(\underset{t=0}{\times}\left(B_{t} /\left(Z_{i t} Z_{j t}\right)\right)\right)\right] \\
& =c \mathbb{E}\left[\tilde{\mu}_{n}^{\sigma^{i j}}\left(\stackrel{n}{X}_{t=0}^{n}\left(B_{t} /\left(Z_{i t} Z_{j t} \prod_{(u, v) \in \Lambda_{i, j, n}^{c}} \mathrm{e}^{\eta_{u, v}\left(\psi_{i-u, t-v}+\psi_{j-u, t-v}\right)}\right)\right)\right)\right]
\end{aligned}
$$

for any $\mu_{n}^{\mathbf{X}^{i j}}$-continuity Borel set $B=\mathrm{X}_{t=0}^{n} B_{t} \in[-\infty, \infty]^{n+1} \backslash\{\mathbf{0}\}$ bounded away from $\mathbf{0}$, $\Lambda_{i, j, n}$ is equal to $\Lambda_{\mathbf{i}, \mathbf{j}, \mathbf{t}}$ as defined in (4.5) with $\mathbf{i}=(i, \ldots, i), \mathbf{j}=(j, \ldots, j), \mathbf{t}=(0, \ldots, n)$, and $\tilde{\mu}_{n}^{\sigma^{i j}}$ is the limit measure of the regularly varying vector

$$
\left(\prod_{(u, v) \in \Lambda_{i, j, n}} \mathrm{e}^{\eta_{u, v}\left(\psi_{i-u, t-v}+\psi_{j-u, t-v}\right)}\right)_{t=0, \ldots, n},
$$

see the proof of Proposition 4.2. The distribution of the tail process of $\left(X_{i t} X_{j t}\right)$ (cf. Section 2.3) is then determined by

$$
\begin{aligned}
\mathbb{P}\left(\left(Y \Theta_{t}^{i j}\right)_{t=0, \ldots, n} \in B\right) & =\lim _{x \rightarrow \infty} \frac{\mathbb{P}\left(\left(X_{i t} X_{j t} / x\right)_{t=0, \ldots, n} \in B,\left|X_{i 0} X_{j 0}\right| / x>1\right)}{\mathbb{P}\left(\left|X_{i 0} X_{j 0}\right| / x>1\right)} \\
& =\frac{\mu_{n}^{\mathbf{X}^{i j}}\left(B \cap\left([-\infty, \infty] \backslash[-1,1] \times[-\infty, \infty]^{n}\right)\right)}{\mu_{n}^{\mathbf{X}^{i j}}\left([-\infty, \infty] \backslash[-1,1] \times[-\infty, \infty]^{n}\right)} .
\end{aligned}
$$

The concrete forms of $\tilde{\mu}_{n}^{\sigma^{i j}}$, hence of $\mu_{n}^{\mathbf{X}^{i j}}$, now depend on whether (4.1) or (4.2) holds.

We first assume (4.1). Note that $\Lambda_{i, j, n}=\bigcup_{t=0}^{n} \Lambda_{i, j}^{(t)}$, where $\Lambda_{i, j}^{(t)}=\left\{(u, v): \psi_{i-u, t-v}+\right.$ $\left.\psi_{j-u, t-v}=\psi^{i j}\right\}$. Indeed, we easily see that $\Lambda_{i, j}^{(t)}=\Lambda_{i, j}^{(0)}+(0, t), t=1, \ldots, n$. We apply Proposition B.3(ii) to derive the specific form of the limit measure $\tilde{\mu}_{n}^{\sigma^{i j}}$ of (4.10). Each component of this vector contains $\left|\Lambda_{i, j}^{(0)}\right|$ factors with maximal exponent $\psi^{i j}$. For the $t$ th component, those are the factors $\exp \left(\eta_{u, v}\left(\psi_{i-u, t-v}+\psi_{j-u, t-v}\right)\right),(u, v) \in \Lambda_{i j}^{(t)}$. Hence, $p_{\text {eff }}=\left|\Lambda_{i, j}^{(0)}\right|$ and $P_{\text {eff }}=\left\{\Lambda_{i, j}^{(0)}+(0, t), t=0, \ldots, n\right\}$. By (B.8), the measure $\tilde{\mu}_{n}^{\sigma^{i j}}$, up to a constant multiple, is 
given by

$$
\begin{aligned}
\tilde{\mu}_{n}^{\sigma^{i j}}(B)= & c \sum_{s=0}^{n} \int_{0}^{\infty} \mathbb{P}\left(\left(\mathbf{1}\left(\psi_{i-u, t-v}+\psi_{j-u, t-v}=\psi^{i j} \forall(u, v) \in \Lambda_{i, j}^{(s)}\right) z^{\psi^{i j}}\right.\right. \\
& \left.\left.\times \prod_{(u, v) \in \Lambda_{i, j, n} \backslash \Lambda_{i, j}^{(s)}} \mathrm{e}^{\eta_{u, v}\left(\psi_{i-u, t-v}+\psi_{j-u, t-v}\right)}\right)_{0 \leq t \leq n} \in B\right) v_{\alpha}(d z) \\
= & c \sum_{s=0}^{n} \int_{0}^{\infty} \mathbb{P}\left(\left(\mathbf{1}(t=s) z^{\psi^{i j}} \prod_{(u, v) \in \Lambda_{i, j, n} \backslash \Lambda_{i, j}^{(s)}} \mathrm{e}^{\eta_{u, v}\left(\psi_{i-u, t-v}+\psi_{j-u, t-v}\right)}\right)_{0 \leq t \leq n} \in B\right) \\
& \times v_{\alpha}(d z),
\end{aligned}
$$

where $v_{\alpha}(d x)=\alpha x^{-\alpha-1} d x$. The $s$ th measure in the sum above is concentrated on the $s$ th axis. Therefore the limit measure $\tilde{\mu}_{n}^{\sigma^{i j}}$ is concentrated on the axes. By (4.9), this implies that $\mu_{n}^{\mathbf{X}^{i j}}$ is concentrated on the axes as well. Therefore $\mu_{n}^{\mathbf{X}^{i j}}\left(B \cap([-\infty, \infty] \backslash[-1,1]) \times[-\infty, \infty]^{n}\right)=0$ as soon as one $B_{i}, 1 \leq i \leq n$, in $B=\mathrm{X}_{i=0}^{n} B_{i}$ is bounded away from 0 . With (4.11) this gives $Y \Theta_{t}^{i j}=0$ a.s. for $t \geq 1$ and therefore $\Theta_{t}^{i j}=0$ a.s. for $t \geq 1$. The law of $\Theta_{0}^{i j}$ follows from the univariate Breiman lemma.

Next assume (4.2). By Proposition B.3(i), the vague limit measure $\tilde{\mu}_{n}^{\sigma^{i j}}$ is up to a constant given by

$$
\begin{aligned}
\tilde{\mu}_{n}^{\sigma^{i j}}(B)= & \sum_{(u, v) \in \Lambda_{i, j, n}} \int_{0}^{\infty} \mathbb{P}\left(\left(\mathbf{1}\left((u, v) \in \Lambda_{i, j}^{(t)}\right) z^{\psi^{i j}}\right.\right. \\
& \times \prod_{\substack{(\tilde{u}, \tilde{v}) \in \Lambda_{i, j, n} \\
(\tilde{u}, \tilde{v}) \neq(u, v)}} \mathrm{e}^{\left.\left(\psi_{\left.i-\tilde{u}, t-\tilde{v}+\psi_{j-\tilde{u}, t-\tilde{v})}\right) \eta_{\tilde{u}, \tilde{v}}}\right)_{t=0, \ldots, n} \in B\right) v_{\alpha}(d z) .}
\end{aligned}
$$

For sets $B$ such that $B \cap\left(\{0\} \times[-\infty, \infty]^{n}\right)=\varnothing$ it suffices thereby to sum only over $(u, v) \in$ $\Lambda_{i, j}^{(0)}$ instead over all $(u, v) \in \Lambda_{i, j, n}=\bigcup_{t=0}^{n} \Lambda_{i, j}^{(t)}$. For these sets, we have by Breiman's lemma (cf. (4.9)),

$$
\begin{aligned}
\mu_{n}^{\mathbf{X}^{i j}}(B) / c= & \sum_{(u, v) \in \Lambda_{i, j}^{(0)}} \int_{0}^{\infty} \mathbb{P}\left(\left(\mathbf{1}\left((u, v) \in \Lambda_{i, j}^{(t)}\right) z^{\psi^{i j}}\right.\right. \\
& \left.\left.\times \prod_{(\tilde{u}, \tilde{v}) \neq(u, v)} \mathrm{e}^{\left(\psi_{i-\tilde{u}, t-\tilde{v}}+\psi_{j-\tilde{u}, t-\tilde{v})}\right) \eta_{\tilde{u}, \tilde{v}}} Z_{i t} Z_{j t}\right)_{t=0, \ldots, n} \in B\right) v_{\alpha}(d z) \\
= & \sum_{(u, v) \in \Lambda_{i, j}^{(0)}} \int_{0}^{\infty} \mathbb{P}\left(\left(\mathbf{1}\left((u, v) \in \Lambda_{i, j}^{(t)}\right) z^{\psi^{i j}} X_{i t} X_{j t} \mathrm{e}^{-\psi^{i j}} \eta_{u, v}\right)_{t=0, \ldots, n} \in B\right) v_{\alpha}(d z),
\end{aligned}
$$


where we used that if $(u, v) \in \Lambda_{i, j}^{(t)}$, then

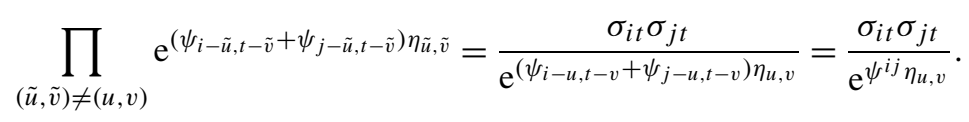

Fubini's theorem and a substitution finally simplify this expression to

$$
\begin{aligned}
& \sum_{(u, v) \in \Lambda_{i, j}^{(0)}} \mathbb{E}\left[\int_{0}^{\infty} \mathbf{1}\left(\left(\mathbf{1}\left((u, v) \in \Lambda_{i, j}^{(t)}\right) z^{\psi^{i j}} X_{i t} X_{j t} \mathrm{e}^{-\psi^{i j} \eta_{u, v}}\right)_{t=0, \ldots, n} \in B\right) v_{\alpha}(d z)\right] \\
& =\sum_{(u, v) \in \Lambda_{i, j}^{(0)}} \mathbb{E}\left[\int_{0}^{\infty} \mathbf{1}\left(\left(\mathbf{1}\left((u, v) \in \Lambda_{i, j}^{(t)}\right) y \frac{X_{i t} X_{j t}}{\left|X_{i 0} X_{j 0}\right|}\right)_{t=0, \ldots, n} \in B\right)\right. \\
& \left.\quad \times\left|X_{i 0} X_{j 0}\right|^{\alpha / \psi^{i j}} \mathrm{e}^{-\alpha \eta_{u, v}} v_{\frac{\alpha}{\psi^{i j}}}(d y)\right] .
\end{aligned}
$$

Note that the range of the inner integral in the last expression can be changed from $(0, \infty)$ to $(1, \infty)$, if $B \cap[-1,1] \times[-\infty, \infty]^{n}=\varnothing$. Therefore, by writing

$$
\tilde{B}_{0}=B_{0} \backslash[-1,1], \quad \tilde{B}_{t}=B_{t}, \quad t \geq 1, \quad \tilde{B}={\underset{X}{t=0}}^{n} \tilde{B}_{t},
$$

we get from (4.11) that

$$
\begin{aligned}
& P\left(\left(Y \Theta_{t}^{i j}\right)_{t=0, \ldots, n} \in B\right) \\
& =\frac{\mu_{n}^{\mathbf{X}^{i j}}(\tilde{B})}{\mu_{n}^{\mathbf{X}^{i j}}\left(([-\infty, \infty] \backslash[-1,1]) \times[-\infty, \infty]^{n}\right)} \\
& =\frac{\sum_{(u, v) \in \Lambda_{i, j}^{(0)}} \mathbb{E}\left[\int_{1}^{\infty} \mathbf{1}\left(\left(\mathbf{1}\left((u, v) \in \Lambda_{i, j}^{(t)}\right) y \frac{X_{i t} X_{j t}}{\left|X_{i 0} X_{j 0}\right|}\right)_{t=0, \ldots, n} \in B\right)\left|X_{i 0} X_{j 0}\right|^{\alpha / \psi^{i j}} \mathrm{e}^{\left.-\alpha \eta_{u, v} \nu_{\frac{\alpha}{\psi^{i j}}}(d y)\right]}\right.}{\sum_{(u, v) \in \Lambda_{i, j}^{(0)}} \mathbb{E}\left[\left|X_{i 0} X_{j 0}\right|^{\alpha / \psi^{i j}} \mathrm{e}^{\left.-\alpha \eta_{u, v}\right]}\right.} \\
& =\sum_{(u, v) \in \Lambda_{i, j}^{(0)}} \frac{1}{\left|\Lambda_{i, j}^{(0)}\right|} \frac{\mathbb{E}\left[\mathbf{1}\left(\left(\mathbf{1}\left((u, v) \in \Lambda_{i, j}^{(t)}\right) Y \frac{X_{i t} X_{j t}}{\left|X_{i 0} X_{j 0}\right|}\right)_{t=0, \ldots, n} \in B\right)\left|X_{i 0} X_{j 0}\right|^{\alpha / \psi^{i j}}\right]}{\mathbb{E}\left[\left|X_{i 0} X_{j 0}\right|^{\alpha / \psi^{i j}}\right]},
\end{aligned}
$$

where $Y$ is a Pareto $\left(\alpha / \psi^{i j}\right)$ random variable, independent of all other random variables in the expression. For the last equation, we expanded both numerator and denominator by multiplying with $\mathbb{E}\left(e^{\alpha \eta_{u, v}}\right)$, noting that for $(u, v) \in \Lambda_{i, j}^{(0)}$ the random variable $e^{\alpha \eta_{u, v}}$ is independent both of the

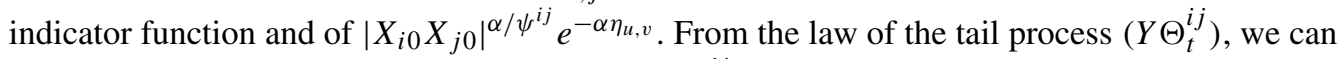
now see that the law of the spectral tail process $\left(\Theta_{t}^{i j}\right)$ satisfies (4.8). 


\subsection{Infinite variance stable limit theory for the stochastic volatility model and its product processes}

In the following result, we provide central limit theory with infinite variance stable limits for the sums $S_{i j}$; see (2.3).

Theorem 4.6. We consider the stochastic volatility model (2.1) and assume the special form of $\left(\sigma_{i t}\right)$ given in (2.7) with $\psi=1$. For given $(i, j)$, define a sequence $\left(b_{n}\right)$ such that $n \mathbb{P}\left(\left|X_{i 0} X_{j 0}\right|>\right.$ $\left.b_{n}\right) \rightarrow 1$ as $n \rightarrow \infty$. Assume the following conditions:

1. The conditions of Proposition 4.4 hold, ensuring that $\mathbb{E}\left[|Z|^{\alpha / \psi^{i j}+\varepsilon}\right]<\infty$ for some $\varepsilon>0$ and $\left(X_{i t} X_{j t}\right)$ is regularly varying with index $\alpha / \psi^{i j}$ and spectral tail process $\left(\Theta_{h}^{i j}\right)$.

2. $\left(\sigma_{i t} \sigma_{j t}\right)$ is $\alpha$-mixing with rate function $\left(\alpha_{h}\right)$ and there exists $\delta>0$ such that $\alpha_{n}=o\left(n^{-\delta}\right)$.

3. Either:

(i) $\alpha / \psi^{i j}<1$, or

(ii) $i \neq j, \alpha / \psi^{i j} \in[1,2)$ and $Z$ is symmetric, or

(iii) $i=j, \alpha / \psi^{i i}=\alpha / 2 \in(1,2)$ and the mixing rate in (2) satisfies $\sup _{n} n \sum_{h=r_{n}}^{\infty} \alpha_{h}<$ $\infty$ for some integer sequence $\left(r_{n}\right)$ such that $n r_{n} / b_{n}^{2} \rightarrow 0$ as $n \rightarrow \infty$.

Then

$$
b_{n}^{-1}\left(S_{i j}-c_{n}\right) \stackrel{d}{\rightarrow} \xi_{i j, \alpha / \psi^{i j}}
$$

where $\xi_{i j, \alpha / \psi^{i j}}$ is a totally skewed to the right $\alpha / \psi^{i j}$-stable random variable and

$$
c_{n}= \begin{cases}n \mathbb{E}\left[X^{2}\right], & i=j \text { and } \alpha \in(2,4), \\ 0, & i \neq j \text { or } \alpha / \psi^{i j}<1 .\end{cases}
$$

Remark 4.7. 1. If $\left(\alpha_{h}\right)$ decays at an exponential rate, one can choose $r_{n}=C \log n$ for a sufficiently large constant $C$. Then $\sup _{n} n \sum_{h=r_{n}}^{\infty} \alpha_{h}<\infty$ and $n r_{n} / b_{n}^{2} \rightarrow 0$ hold. These conditions are also satisfied if $\alpha_{h} \leq c n^{-(1+\gamma)}$ for some $\gamma>0, r_{n}=C n^{\xi}$ for some $\xi>0$ and $1 / \gamma \leq \xi<2 \psi^{i j} / \alpha-1$.

2. The sequence $\left(X_{i t} X_{j t}\right)$ inherits $\alpha$-mixing from $\left(\sigma_{i t} \sigma_{j t}\right)$; see Remark 3.5.

3. It is possible to prove joint convergence for $1 \leq i, j \leq p$ in (4.12). Due to different tail behavior for distinct $(i, j)$ the normalizing sequences $\left(b_{n}\right)=\left(b_{n}^{i j}\right)$ typically increase to infinity at different rates. Then it is only of interest to consider the joint convergence of those $S_{i j}$ whose summands $X_{i t} X_{j t}$ have the same tail index $\alpha / \psi^{i j}$. More precisely, it suffices to consider those $S_{i j}$ with the property that $X_{i t} X_{j t}$ is tail-equivalent to $X_{i t}^{2}$. The joint convergence follows in a similar way as in the proof below, by observing that Theorem A.1 is a multivariate limit result. The joint limit of $S_{i j}$ in (4.12) with equivalent tails of index $\tilde{\alpha}$ (say) is jointly $\tilde{\alpha}$-stable with possible dependencies in the limit vector.

4. The strongest normalization is needed for $S_{i}=S_{i i}$. Recall that the summands $X_{i t}^{2}$ of $S_{i}$ are regularly varying with index $\alpha / 2$, that is, $\psi^{i i}=2$. Let $\left(a_{n}\right)$ be such that $n \mathbb{P}\left(|X|>a_{n}\right) \rightarrow 1$. 
Under the conditions of Theorem 4.6, we have that $a_{n}^{-2}\left(S_{i}-c_{n}\right) \stackrel{d}{\rightarrow} \xi_{i, \alpha / 2}, i=1, \ldots, p$ for a jointly $\alpha / 2$-stable limit. If $\alpha / 2<\alpha / \psi^{i j}$ for some $i \neq j$, then $b_{n} / a_{n}^{2} \rightarrow 0$, hence $a_{n}^{-2} S_{i j} \stackrel{\mathbb{P}}{\rightarrow} 0$. It is possible that $X_{i t} X_{j t}$ is regularly varying with index $\alpha / 2$ but nevertheless $b_{n} / a_{n}^{2} \rightarrow 0$; see Example 4.8 which deals with the case $\mathbb{E}\left[\mathrm{e}^{\alpha \eta}\right]=\infty$.

Proof. We apply Theorem A.1 to the sequence $\left(X_{i t} X_{j t}\right)$, cf. also Remark A.2.

(1) The regular variation condition on $\left(X_{i t} X_{j t}\right)$ with index $\alpha / \psi^{i j}$ is satisfied by assumption. Moreover, $\Theta_{h}=0$ for sufficiently large $h$; see Remark 4.5.

(2) The assumption about the mixing coefficients in condition (2) implies that for a sufficiently small $\varepsilon \in(0,1)$ and $m_{n}=n^{1-\varepsilon}$ there exists an integer sequence $l_{n}=o\left(m_{n}\right)$ such that $k_{n} \alpha_{l_{n}} \rightarrow 0$. For this choice of $m_{n}$ and $l_{n}$, the proof of the mixing condition for the sums of the truncated variables

$$
\underline{S}_{i j}=\sum_{t=1}^{n} X_{i t} X_{j t} \mathbf{1}\left(\left|X_{i t} X_{j t}\right|>\varepsilon b_{n}\right)
$$

is now analogous to the proof of the corresponding property in Theorem 3.3.

(3) We want to show that

$$
\limsup _{l \rightarrow \infty} \limsup _{n \rightarrow \infty} \sum_{t=l}^{m_{n}} \mathbb{P}\left(\left|X_{i t} X_{j t}\right|>b_{n},\left|X_{i 0} X_{j 0}\right|>b_{n}\right)=0
$$

for $m_{n}=n^{1-\varepsilon}$ as above. Write

$$
\sigma_{i t} \sigma_{j t}=\prod_{(m, n)} \exp \left(\left(\psi_{i-m, t-n}+\psi_{j-m, t-n}\right) \eta_{m, n}\right)
$$

and set $\Lambda_{\varepsilon, t}=\left\{(m, n): \psi_{i-m, t-n}+\psi_{j-m, t-n} \geq 8^{-1} \psi^{i j} \varepsilon\right\}, t \in \mathbb{Z}$. Without loss of generality, we assume that $l$ is so large that $\Lambda_{\varepsilon, t} \cap \Lambda_{\varepsilon, 0}$ is empty for all $t \geq l$. Then write for $t \geq l$,

$$
\sigma_{i t} \sigma_{j t}=\sigma_{i t, j t, \Lambda_{\varepsilon, t}} \cdot \sigma_{i t, j t, \Lambda_{\varepsilon, 0}} \cdot \sigma_{i t, j t, \Lambda_{\varepsilon, 0, t}^{c},} \quad \sigma_{i 0} \sigma_{j 0}=\sigma_{i 0, j 0, \Lambda_{\varepsilon, 0}} \cdot \sigma_{i 0, j 0, \Lambda_{\varepsilon, t}} \cdot \sigma_{i 0, j 0, \Lambda_{\varepsilon, 0, t}^{c}},
$$

where

$$
\sigma_{i t_{1}, j t_{1}, \Lambda_{\varepsilon, t_{2}}}=\prod_{(m, n) \in \Lambda_{\varepsilon, t_{2}}} \exp \left(\left(\psi_{i-m, t_{1}-n}+\psi_{j-m, t_{1}-n}\right) \eta_{m, n}\right) .
$$

We conclude that $\left(\sigma_{i t, j t, \Lambda_{\varepsilon, t}}, \sigma_{i t, j t, \Lambda_{\varepsilon, 0}}, \sigma_{i 0, j 0, \Lambda_{\varepsilon, 0}}, \sigma_{i 0, j 0, \Lambda_{\varepsilon, t}}\right)$ and $\left(\sigma_{i t, j t, \Lambda_{\varepsilon, 0, t}^{c}}, \sigma_{i 0, j 0, \Lambda_{\varepsilon, 0, t}^{c}}\right)$ are independent. We have

$$
\begin{aligned}
& \mathbb{P}\left(\left|X_{i t} X_{j t}\right|>b_{n},\left|X_{i 0} X_{j 0}\right|>b_{n}\right) \\
& \leq \mathbb{P}\left(\max \left(\left|Z_{i 0} Z_{j 0}\right|,\left|Z_{i t} Z_{j t}\right|\right) \max \left(\sigma_{i t, j t, \Lambda_{\varepsilon, 0, t}^{c}}, \sigma_{i 0, j 0, \Lambda_{\varepsilon, 0, t}^{c}}\right)\right. \\
& \left.\times \min \left(\sigma_{i 0, j 0, \Lambda_{\varepsilon, 0}} \sigma_{i 0, j 0, \Lambda_{\varepsilon, t}}, \sigma_{i t, j t, \Lambda_{\varepsilon, t}} \sigma_{i t, j t, \Lambda_{\varepsilon, 0}}\right)>b_{n}\right) .
\end{aligned}
$$




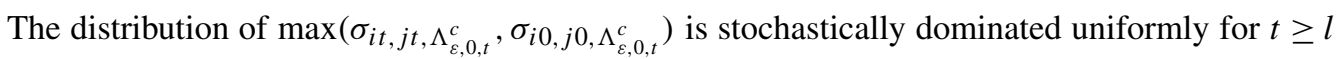
by a distribution which has moment of order $8 \alpha /\left(\psi^{i j} \varepsilon\right)>2 \alpha / \psi^{i j}$. Furthermore,

$$
\begin{aligned}
& \min \left(\sigma_{i 0, j 0, \Lambda_{\varepsilon, 0}} \sigma_{i 0, j 0, \Lambda_{\varepsilon, t}}, \sigma_{i t, j t, \Lambda_{\varepsilon, t}} \sigma_{i t, j t, \Lambda_{\varepsilon, 0}}\right) \\
& \leq \min \left(\prod_{(m, n) \in \Lambda_{\varepsilon, 0} \cup \Lambda_{\varepsilon, t}} \exp \left(\left(\psi_{i-m,-n}+\psi_{j-m,-n}\right)\left(\eta_{m, n}\right)_{+}\right),\right. \\
& \left.\prod_{(m, n) \in \Lambda_{\varepsilon, 0} \cup \Lambda_{\varepsilon, t}} \exp \left(\left(\psi_{i-m, t-n}+\psi_{j-m, t-n}\right)\left(\eta_{m, n}\right)_{+}\right)\right) \\
& \leq \min \left(\prod_{(m, n) \in \Lambda_{\varepsilon, 0}} \exp \left(\psi^{i j}\left(\eta_{m, n}\right)_{+}\right) \prod_{\left(m^{\prime}, n^{\prime}\right) \in \Lambda_{\varepsilon, t}} \exp \left(8^{-1} \psi^{i j} \varepsilon\left(\eta_{m^{\prime}, n^{\prime}}\right)_{+}\right),\right. \\
& \left.\prod_{\left(m^{\prime}, n^{\prime}\right) \in \Lambda_{\varepsilon, t}} \exp \left(\psi^{i j}\left(\eta_{m^{\prime}, n^{\prime}}\right)_{+}\right) \prod_{(m, n) \in \Lambda_{\varepsilon, 0}} \exp \left(8^{-1} \psi^{i j} \varepsilon\left(\eta_{m, n}\right)_{+}\right)\right) \\
& \leq \min \left(\prod_{(m, n) \in \Lambda_{\varepsilon, 0}} \exp \left(\left(\psi^{i j}+8^{-1} \psi^{i j} \varepsilon\right)\left(\eta_{m, n}\right)_{+}\right)\right. \\
& \left.\prod_{(m, n) \in \Lambda_{\varepsilon, t}} \exp \left(\left(\psi^{i j}+8^{-1} \psi^{i j} \varepsilon\right)\left(\eta_{m, n}\right)_{+}\right)\right) \text {. }
\end{aligned}
$$

The right-hand side is regularly varying with index $2 \alpha /\left(\psi^{i j}\left(1+8^{-1} \varepsilon\right)\right)$. A stochastic domination argument and an application of Breiman's lemma show that uniformly for $l \leq t \leq m_{n}$,

$$
\begin{aligned}
m_{n} n \mathbb{P}\left(\left|X_{i t} X_{j t}\right|>b_{n},\left|X_{i 0} X_{j 0}\right|>b_{n}\right) & =n^{2-\varepsilon} o\left(b_{n}^{-2 \alpha /\left(\psi^{i j}\left(1+4^{-1} \epsilon\right)\right)}\right) \\
& =n^{2-\varepsilon} o\left(n^{-2 /\left(1+2^{-1} \varepsilon\right)}\right) \\
& =o(1)
\end{aligned}
$$

which yields (4.13).

(4) We check the vanishing small values condition. For any fixed $\delta$, we write

$$
\begin{aligned}
\overline{X_{i t} X_{j t}} & =X_{i t} X_{j t} \mathbf{1}\left(\left|X_{i t} X_{j t}\right| \leq \delta b_{n}\right), \quad i \neq j, \\
\overline{X_{i t}^{2}} & =X_{i t}^{2} \mathbf{1}\left(X_{i t}^{2} \leq \delta b_{n}\right)-\mathbb{E}\left[X_{i t}^{2} \mathbf{1}\left(X_{i t}^{2} \leq \delta b_{n}\right)\right], \\
\bar{S}_{i j} & =\sum_{t=1}^{n} \overline{X_{i t} X_{j t}}, \quad \bar{S}_{i}=\bar{S}_{i i} .
\end{aligned}
$$


Assume $\alpha / \psi^{i j} \in[1,2), i \neq j$. Then, by symmetry of the random variables $Z_{i t}$ and Karamata's theorem for any $\gamma>0$ as $n \rightarrow \infty, \mathbb{E}\left[\bar{S}_{i j}\right]=0$ and

$$
\begin{aligned}
\mathbb{P}\left(\left|\bar{S}_{i j}\right|>\gamma b_{n}\right) & \leq\left(\gamma b_{n}\right)^{-2} \operatorname{var}\left(\bar{S}_{i j}\right) \\
& =n\left(\gamma b_{n}\right)^{-2} \mathbb{E}\left[\left(\overline{X_{i t} X_{j t}}\right)^{2}\right] \\
& \sim \gamma^{-2} \delta^{2-\alpha},
\end{aligned}
$$

and the right-hand side converges to zero as $\delta \downarrow 0$.

For $i=j$ and $\alpha / \psi^{i i}>1$ we need a different argument. We have by Čebyshev's inequality,

$$
\begin{aligned}
\mathbb{P}\left(\left|\bar{S}_{i}\right|>\gamma b_{n}\right) & \leq \gamma^{-2} b_{n}^{-2} \operatorname{var}\left(\bar{S}_{i}\right) \\
& =\gamma^{-2}\left(n / b_{n}^{2}\right) \sum_{|h|<n}(1-h / n) \operatorname{cov}\left(\overline{X_{i 0}^{2}}, \overline{X_{i h}^{2}}\right) .
\end{aligned}
$$

For $|h| \leq h_{0}$ for any fixed $h_{0},\left(n / b_{n}^{2}\right)\left|\operatorname{cov}\left(\overline{X_{i 0}^{2}}, \overline{X_{i h}^{2}}\right)\right|$ vanishes by letting first $n \rightarrow \infty$ and then $\delta \downarrow 0$. This follows by Karamata's theorem. Standard bounds for the covariance function of an $\alpha$-mixing sequence (see Doukhan [26], page 3) yield

$$
\left(n / b_{n}^{2}\right) \sum_{r_{n} \leq|h|<n}\left|\operatorname{cov}\left(\overline{X_{i 0}^{2}}, \overline{X_{i h}^{2}}\right)\right| \leq c \delta^{2} n \sum_{r_{n} \leq|h|<n} \alpha_{h},
$$

where $r_{n} \rightarrow \infty$ is chosen such that $\sup _{n} n \sum_{r_{n} \leq|h|<\infty} \alpha_{h}<\infty$ and $n r_{n} / b_{n}^{2} \rightarrow 0$. The right-hand side converges to zero by first letting $n \rightarrow \infty$ and then $\delta \downarrow 0$. It remains to show that

$$
I_{n}=\left(n / b_{n}^{2}\right) \sum_{h_{0}<|h| \leq r_{n}}(1-h / n) \operatorname{cov}\left(\overline{X_{i 0}^{2}}, \overline{X_{i h}^{2}}\right)
$$

is asymptotically negligible. We have

$$
\begin{aligned}
\left|I_{n}\right| & \leq\left(n / b_{n}^{2}\right) \sum_{h_{0}<|h| \leq r_{n}} \mathbb{E}\left[X_{i 0}^{2} X_{i h}^{2} \mathbf{1}\left(X_{i 0}^{2} \leq \delta b_{n}, X_{i h}^{2} \leq \delta b_{n}\right)\right]+c n r_{n} / b_{n}^{2} \\
& \leq\left(n / b_{n}^{2}\right) \sum_{h_{0}<|h| \leq r_{n}} \mathbb{E}\left[X_{i 0}^{2} X_{i h}^{2}\right]+o(1),
\end{aligned}
$$

where we used that $n r_{n} / b_{n}^{2} \rightarrow 0$. We will show that the summands on the right-hand side are uniformly bounded by a constant if $h_{0}$ is sufficiently large. Then $\lim _{n \rightarrow \infty} I_{n}=0$.

We observe that by Hölder's inequality,

$$
\begin{aligned}
\mathbb{E}\left[X_{i 0}^{2} X_{i h}^{2}\right] & =c \mathbb{E}\left[\sigma_{i 0}^{2} \sigma_{i h}^{2}\right] \\
& =c \mathbb{E}\left[\mathrm{e}^{2 \sum_{(k, l) \in \Gamma_{\xi}} \psi_{k l}\left(\eta_{i-k,-l}+\eta_{i-k, h-l}\right)} \mathrm{e}^{2 \sum_{(k, l) \notin \Gamma_{\xi}} \psi_{k l}\left(\eta_{i-k,-l}+\eta_{i-k, h-l}\right)}\right] \\
& \leq c\left(\mathbb{E}\left[\mathrm{e}^{2 r \sum_{(k, l) \in \Gamma_{\xi}} \psi_{k l}\left(\eta_{i-k,-l}+\eta_{i-k, h-l}\right)}\right]\right)^{1 / r}\left(\mathbb{E}\left[\mathrm{e}^{2 s \sum_{(k, l) \notin \Gamma_{\xi}} \psi_{k l}\left(\eta_{i-k,-l}+\eta_{i-k, h-l}\right)}\right]\right)^{1 / s},
\end{aligned}
$$


where $\Gamma_{\xi}=\left\{(k, l): \psi_{i k}>\xi\right\}$ for some positive $\xi, s, t$ such that $1 / r+1 / s=1$. Since $\sigma_{i 0}^{2}$ has moments up to order $\alpha / \psi^{i i} \in(1,2)$ and $\left(\eta_{i-k,-l}\right)_{(k, l) \in \Gamma_{\xi}}$ and $\left(\eta_{i-k, h-l}\right)_{(k, l) \in \Gamma_{\xi}}$ are independent for sufficiently large $h$ we can choose $r>1$ close to one such that $\mathbb{E}\left[\mathrm{e}^{2 r \sum_{(k, l) \in \Gamma \xi} \psi_{k l}\left(\eta_{i-k,-l}+\eta_{i-k, h-l}\right)}\right]$ is finite. This implies that we choose $s$ sufficiently large. On the other hand, for fixed $s$ we can make $\xi$ so small that $\mathbb{E}\left[\mathrm{e}^{2 s \sum_{(k, l) \notin \Gamma \xi} \psi_{k l}\left(\eta_{i-k,-l}+\eta_{i-k, h-l}\right)}\right]$ is finite and uniformly bounded for sufficiently large $h$. Fine tuning $\xi$ and $s$, we may conclude that $\lim _{n \rightarrow \infty} I_{n}=0$ as desired.

By Theorem A.1 and Remark A.2 the result now follows; see also the end of the proof of Theorem 3.3 for the form of the resulting limit law.

Example 4.8. We assume that $\mathbb{E}\left[\mathrm{e}^{\alpha \eta}\right]=\infty$, hence $\mathrm{e}^{2 \eta}$ does not have a finite $\alpha / 2$ th moment. Using Lemma B.1(5), calculation shows that for $i \neq j$ with $\psi^{i j}=2$,

$$
\lim _{x \rightarrow \infty} \frac{\mathbb{P}\left(\left|X_{i 0} X_{j 0}\right|>x\right)}{\mathbb{P}\left(X^{2}>x\right)}=0 .
$$

Define $\left(a_{n}\right)$ such that $n \mathbb{P}\left(|X|>a_{n}\right) \rightarrow 1$. We may conclude from (4.14) and Theorem 4.6 that for $i \neq j$ we have $a_{n}^{-2} S_{i j} \stackrel{\mathbb{P}}{\rightarrow} 0$ although both $X_{i 0} X_{j 0}$ and $X^{2}$ are regularly varying with index $\alpha / 2$.

By Theorem 4.6 and Remark 4.7, we conclude that

$$
a_{n}^{-2}\left(S_{i}-c_{n}\right)_{i=1, \ldots, p} \stackrel{d}{\rightarrow}\left(\xi_{i, \alpha / 2}\right)_{i=1, \ldots, p},
$$

where the limit vector consists of $\alpha / 2$-stable components. The spectral tail process $\left(\boldsymbol{\Theta}_{h}\right)_{h \geq 1}$ of the sequence $\mathbf{X}_{t}=\left(X_{1 t}, \ldots, X_{p t}\right)^{\prime}, t=1,2, \ldots$, vanishes. This follows by an argument similar to the proofs of Propositions 4.4 and B.3 under condition (4.1). A similar argument also yields that

$$
\lim _{x \rightarrow \infty} \frac{\mathbb{P}\left(\left|X_{i 0}\right|>x,\left|X_{j 0}\right|>x\right)}{\mathbb{P}(|X|>x)}=0, \quad i \neq j .
$$

Therefore the the distribution of $\boldsymbol{\Theta}_{0}$ is concentrated on the axes and has the same form as $\boldsymbol{\Theta}_{0}^{(2)}$ in (3.6). As in the proof of Theorem 3.3 this implies that the limit random vector in (4.15) has i.i.d. components.

We conclude that the limit theory for $S_{i j}, 1 \leq i, j \leq p$, are very essentially the same in Case (1) and in Case (2) when the additional condition $\mathbb{E}\left[\mathrm{e}^{\alpha \eta}\right]=\infty$ holds.

Example 4.9. Assume that (4.2) holds. We may conclude from Theorem 4.6 that $a_{n}^{-2} S_{i j} \stackrel{\mathbb{P}}{\rightarrow} 0$ for $i \neq j$ if $\psi^{i j}<2$. The crucial difference to the previous case appears when $\psi^{i j}=2$ for some $i \neq j$. In this case, not only the $\left(a_{n}^{-2}\left(S_{i}-c_{n}\right)\right), i=1,2, \ldots$, have totally skewed to the right $\alpha / 2$ stable limits but we also have $a_{n}^{-2} S_{i j} \stackrel{d}{\rightarrow} \xi_{i j, \alpha / 2}$ for non-degenerate $\alpha / 2$-stable $\xi_{i j, \alpha / 2}$. From (4.3), we conclude that if $\psi^{i j}=2$ appears then $\psi^{i^{\prime} j^{\prime}}=2$ for all $\left|i^{\prime}-j^{\prime}\right|=|i-j|$. This means that non-degenerate limits may appear not only on the diagonal of the matrix $a_{n}^{-2}\left(S_{i j}-c_{n}\right)$ but also along full sub-diagonals. 
In this case, the distribution of $\boldsymbol{\Theta}_{0}$ from the spectral tail process of the sequence $\mathbf{X}_{t}=$ $\left(X_{1 t}, \ldots, X_{p t}\right)^{\prime}$ does not have to be concentrated on the axes - in contrast to Example 4.8. This implies that the limiting $\alpha / 2$-stable random variables $\xi_{i, \alpha / 2}, i=1, \ldots, p$, are in general not independent. However, similar to the arguments at the end of the proof of Theorem 3.3, one can show that the distribution of the limiting random vector $\left(\xi_{i, \alpha / 2}\right)_{i=1, \ldots, p}$ is the convolution of distributions of $\alpha / 2$-stable random vectors which concentrate on hyperplanes of $\mathbb{R}^{p}$ of dimension less or equal than $\left|\left\{(m, n): \psi_{m n}=1\right\}\right|$.

\subsection{The eigenvalues of the sample covariance matrix of a multivariate stochastic volatility model}

In this section, we provide some results for the eigenvalues of the sample covariance matrix $\mathbf{X}^{n}\left(\mathbf{X}^{n}\right)^{\prime}$ under the conditions of Theorem 4.6. We introduce the sets

$$
\Gamma_{p}=\left\{(i, j): 1 \leq i, j \leq p \text { such that } \psi^{i j}=2\right\}, \quad \Gamma_{p}^{c}=\{(i, j): 1 \leq i, j \leq p\} \backslash \Gamma_{p}
$$

and let $\left(a_{n}\right)$ be such that $n \mathbb{P}\left(|X|>a_{n}\right) \rightarrow 1$.

Theorem 4.10. Assume that the conditions of Theorem 4.6 hold for $\left(X_{i t}, X_{j t}\right), 1 \leq i, j \leq p$, and $\alpha \in(0,4)$. Then

$$
a_{n}^{-2}\left\|\mathbf{X}^{n}\left(\mathbf{X}^{n}\right)^{\prime}-\widetilde{\mathbf{X}}^{n}\right\|_{2} \stackrel{\mathbb{P}}{\rightarrow} 0, \quad n \rightarrow \infty
$$

where $\widetilde{\mathbf{X}}^{n}$ is a $p \times p$ matrix with entries

$$
\widetilde{X}_{i j}=\sum_{t=1}^{n} X_{i t} X_{j t} \mathbf{1}\left((i, j) \in \Gamma_{p}\right), \quad 1 \leq i, j \leq p .
$$

Moreover, if $\mathbb{E}\left[\mathrm{e}^{\alpha \eta}\right]=\infty$ we also have

$$
a_{n}^{-2}\left\|\mathbf{X}^{n}\left(\mathbf{X}^{n}\right)^{\prime}-\operatorname{diag}\left(\mathbf{X}^{n}\left(\mathbf{X}^{n}\right)^{\prime}\right)\right\|_{2} \stackrel{\mathbb{P}}{\rightarrow} 0, \quad n \rightarrow \infty
$$

Proof. We have

$$
a_{n}^{-4}\left\|\mathbf{X}^{n}\left(\mathbf{X}^{n}\right)^{\prime}-\widetilde{\mathbf{X}}^{n}\right\|_{2}^{2} \leq \sum_{(i, j) \in \Gamma_{p}^{c}}\left(a_{n}^{-2} S_{i j}\right)^{2} .
$$

For $(i, j) \in \Gamma_{p}^{c}$ we have $i \neq j$ and the sequence $\left(X_{i t} X_{j t}\right)$ is regularly varying with index $\alpha / \psi^{i j}>$ $\alpha / 2$. In view of Theorem 4.6 the right-hand side converges to zero in probability.

In the case when $\mathbb{E}\left[\mathrm{e}^{\alpha \eta}\right]=\infty$, we learned in Example 4.8 that $a_{n}^{-2} S_{i j} \stackrel{\mathbb{P}}{\rightarrow} 0$ whenever $i \neq j$. This concludes the proof.

For any $p \times p$ non-negative definite matrix $\mathbf{A}$ write $\lambda_{i}(\mathbf{A}), i=1, \ldots, p$, for its eigenvalues and $\lambda_{(1)}(\mathbf{A}) \geq \cdots \geq \lambda_{(p)}(\mathbf{A})$ for their ordered values. For the eigenvalues of $\mathbf{X}^{n}\left(\mathbf{X}^{n}\right)^{\prime}$, we keep the previous notation $\left(\lambda_{i}\right)$. 
Corollary 4.11. Assume the conditions of Theorem 4.10 and $\alpha \in(0,4) \backslash\{2\}$. Then

$$
a_{n}^{-2} \max _{i=1, \ldots, p}\left|\lambda_{(i)}-\lambda_{(i)}\left(\widetilde{\mathbf{X}}^{n}\right)\right| \stackrel{\mathbb{P}}{\rightarrow} 0
$$

and

$$
\begin{aligned}
& a_{n}^{-2}\left(\lambda_{(i)}-n \mathbb{E}\left[X^{2}\right] 1(\alpha \in(2,4))\right)_{i=1, \ldots, p} \\
& \quad \stackrel{d}{\rightarrow}\left(\lambda_{(i)}\left(\left(\xi_{k l, \alpha / 2} \mathbf{1}\left((k, l) \in \Gamma_{p}\right)\right)_{1 \leq k, l \leq p}\right)\right)_{i=1, \ldots, p},
\end{aligned}
$$

where $\left(\xi_{i j, \alpha / 2}\right)_{(i, j) \in \Gamma_{p}}$ are jointly $\alpha / 2$-stable (possibly degenerate for $i \neq j$ ) random variables. Moreover, in the case when $\mathbb{E}\left[\mathrm{e}^{\alpha \eta}\right]=\infty$ we have

$$
a_{n}^{-2}\left(\lambda_{(i)}-n \mathbb{E}\left[X^{2}\right] 1(\alpha \in(2,4))\right)_{i=1, \ldots, p} \stackrel{d}{\rightarrow}\left(\xi_{(i), \alpha / 2}\right)_{i=1, \ldots, p},
$$

where $\left(\xi_{i, \alpha / 2}\right)_{i=1, \ldots, p}$ are i.i.d. totally skewed to the right $\alpha / 2$-stable random variables with order statistics $\xi_{(1), \alpha / 2} \geq \cdots \geq \xi_{(p), \alpha / 2}$.

Proof. Relation (4.16) is an immediate consequence of Theorem 4.10 and Weyl's inequality; see Bhatia [9]. We conclude from Theorem 4.6 and Remark 4.7(3) that

$$
a_{n}^{-2}\left(S_{i j}-n \mathbb{E}\left[X^{2}\right] \mathbf{1}(\alpha \in(2,4))\right)_{(i, j) \in \Gamma_{p}} \stackrel{d}{\rightarrow}\left(\xi_{i j, \alpha / 2}\right)_{(i, j) \in \Gamma_{p}}
$$

Then (4.17) follows. Relation (4.18) is a special case of (4.17). If $\mathbb{E}\left[\mathrm{e}^{\alpha \eta}\right]=\infty$ then, in view of Example 4.8, only the diagonal elements in (4.19) have non-degenerate i.i.d. $\alpha / 2$-stable limits.

\section{Some conclusions}

By virtue of this corollary and in view of Section 3.3 the results for the eigenvalues in Case (1) and in Case (2) when $\mathbb{E}\left[\mathrm{e}^{\alpha \eta}\right]=\infty$ are very much the same. Moreover, the results in Section 3.4 remain valid in the latter case.

If (4.2) holds, Case (2) is quite different from Case (1); see Example 4.9. In this case, not only the diagonal of the matrix $\mathbf{X}^{n}\left(\mathbf{X}^{n}\right)^{\prime}$ determines the asymptotic behavior of its eigenvalues and eigenvectors. Indeed, if $\psi^{i j}=2$ for some $i \neq j$, then at least two sub-diagonals of $\mathbf{X}^{n}\left(\mathbf{X}^{n}\right)^{\prime}$ have non-degenerate $\alpha / 2$-limits and these sub-diagonals together with the diagonal determine the asymptotic behavior of the eigenspectrum. The limiting diagonal elements are dependent in contrast to Case (1). This fact and the presence of sub-diagonals are challenges if one wants to calculate the limit distributions of the eigenvalues and eigenvectors.

\section{Simulations and data example}

In this section, we illustrate the behavior of sample covariance matrices for moderate sample sizes for the models discussed in Sections 3 and 4 and we compare them with a real-life data example. 


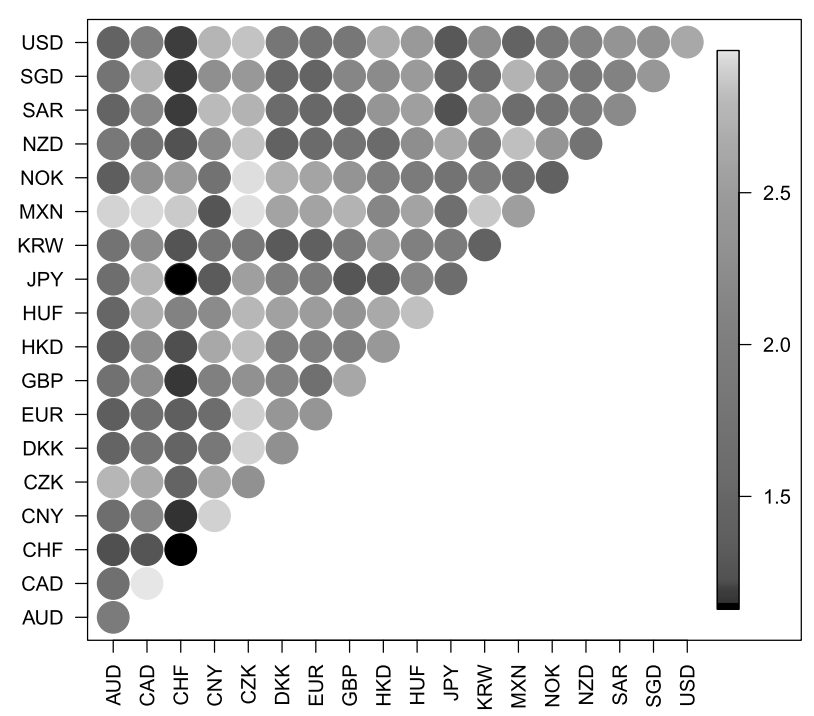

Figure 1. Estimated tail indices of cross products for the FX rates of 18 currencies against SEK. The indices are derived by Hill estimators with threshold equal to the $97 \%$-quantile of $n=1567$ observations.

These data consist of 1567 daily log-returns of foreign exchange (FX) rates from 18 currencies against the Swedish Kroner (SEK) from January 4th 2010 to April 1st 2016, as made available by the Swedish National Bank. To start with, the Hill estimators of the tail indices $\alpha_{i j}, 1 \leq i, j, \leq 18$, of the cross products $X_{i t} X_{j t}, 1 \leq i, j, \leq 18$, are visualized in Figure 1. In particular, the Hill estimators on the diagonal (corresponding to the series $X_{i t}^{2}, 1 \leq i \leq 18$ ) of the values $\alpha_{i} / 2$, where $\alpha_{i}$ is the tail index of the $i$ th currency, are of similar size although not identical. Even if all series had the same tail index the Hill estimator exhibits high statistical uncertainty which even increases for serially dependent data, cf. Drees [27]. A way to make the data more homogeneous in their tails is to rank-transform their marginals to the same distribution. We do, however, refrain from such a transformation to keep the correlation structure of the original data unchanged.

It is clearly visible that some off-diagonal components of the matrix have an estimated tail index which is comparable to the on-diagonal elements. This implies that the tails of the corresponding off-diagonal entries $S_{i j}, i \neq j$, of the sample covariance matrix may be of a similar magnitude as the on-diagonal entries $S_{i}$. This is in stark contrast to the asymptotic behavior of the models analyzed in Section 3.

Figure 2(a) shows the ordered eigenvalues of the sample covariance matrix (normalized by its trace) and the eigenvector of the FX rate data corresponding to the largest eigenvalue. There exists a notable spectral gap between the largest and second largest eigenvalues and the unit eigenvector corresponding to the largest eigenvector has all positive and non-vanishing components. For comparison and to illustrate the variety of the models discussed above, we also plot corresponding realizations of three model specifications from Sections 3 and 4 . In all cases, we choose $p=18$ and $n=1567$ in accordance with the data example. We assume throughout a 

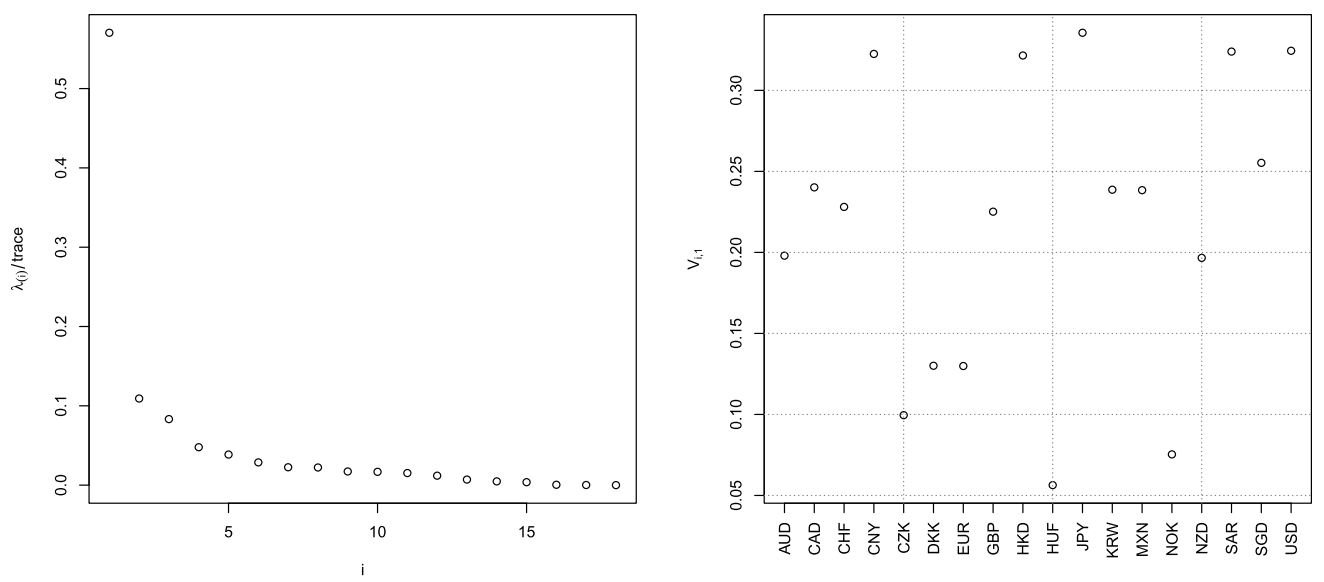

(a)
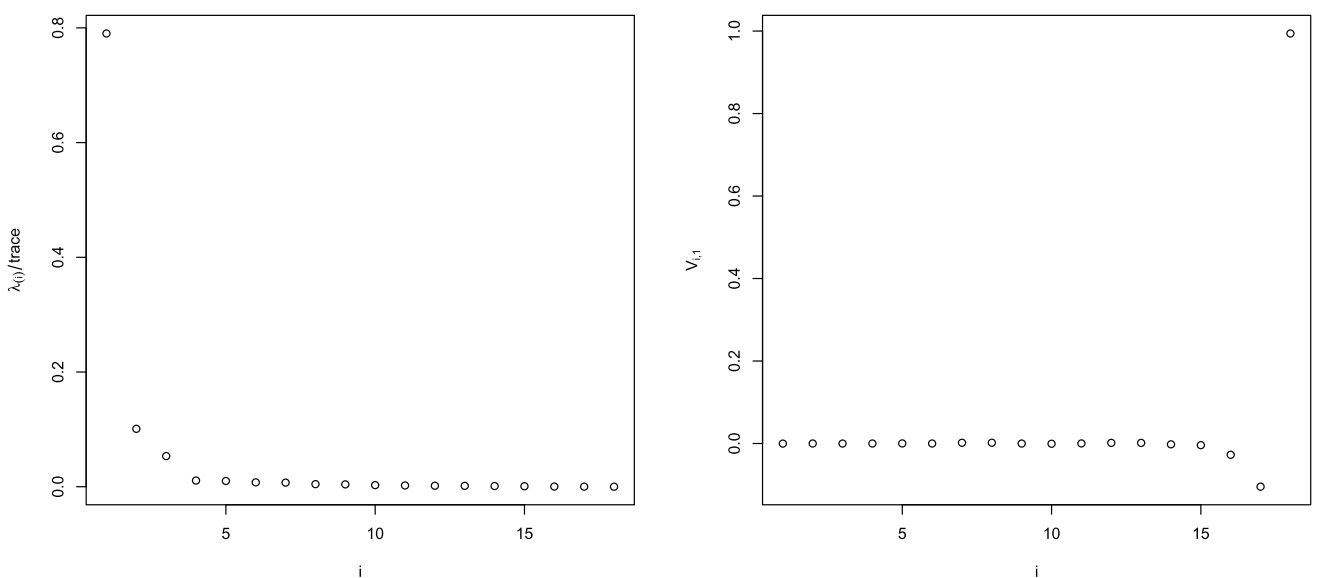

(b)

Figure 2. Normalized and ordered eigenvalues (left) and eigenvector corresponding to largest eigenvalue (right) of real and simulated data, with $n=1567, p=18$. (a) Based on FX rate data of 18 foreign currencies against SEK. (b) Based on a stochastic volatility model with heavy-tailed innovation sequence. (c) Based on a stochastic volatility model with heavy-tailed volatility sequence that satisfies assumptions of Example 4.8. (d) Based on a stochastic volatility model with heavy-tailed volatility sequence that satisfies assumptions of Example 4.9.

moving average structure in the $\log$-volatility process $\log \sigma_{i t}$ in (2.1). More specifically,

$$
\sigma_{i t}=\exp \left(\sum_{k=1}^{18} \eta_{i-k, t}\right), \quad 1 \leq i \leq 18, t \in \mathbb{Z} .
$$

In accordance with the model properties discussed in Section 3, we first assume i.i.d. standard Gaussian $\eta_{i, t}$ and i.i.d. $Z_{i t}$ with a Student- $t$ distribution with $t=3$ degrees of freedom. Fig- 

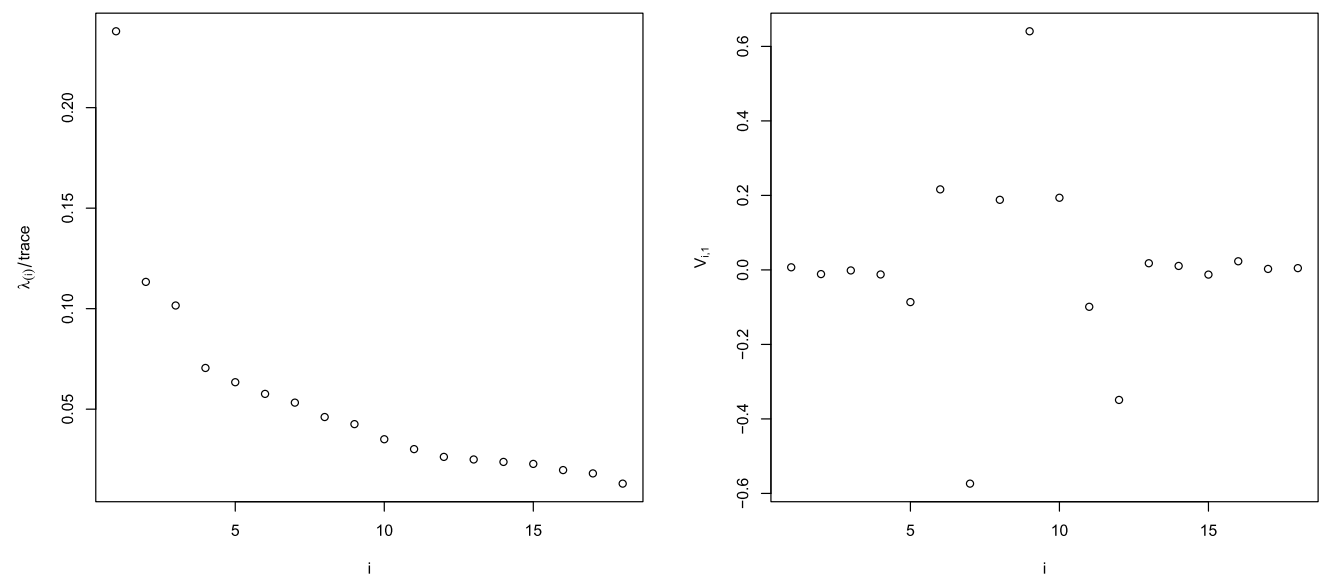

(c)
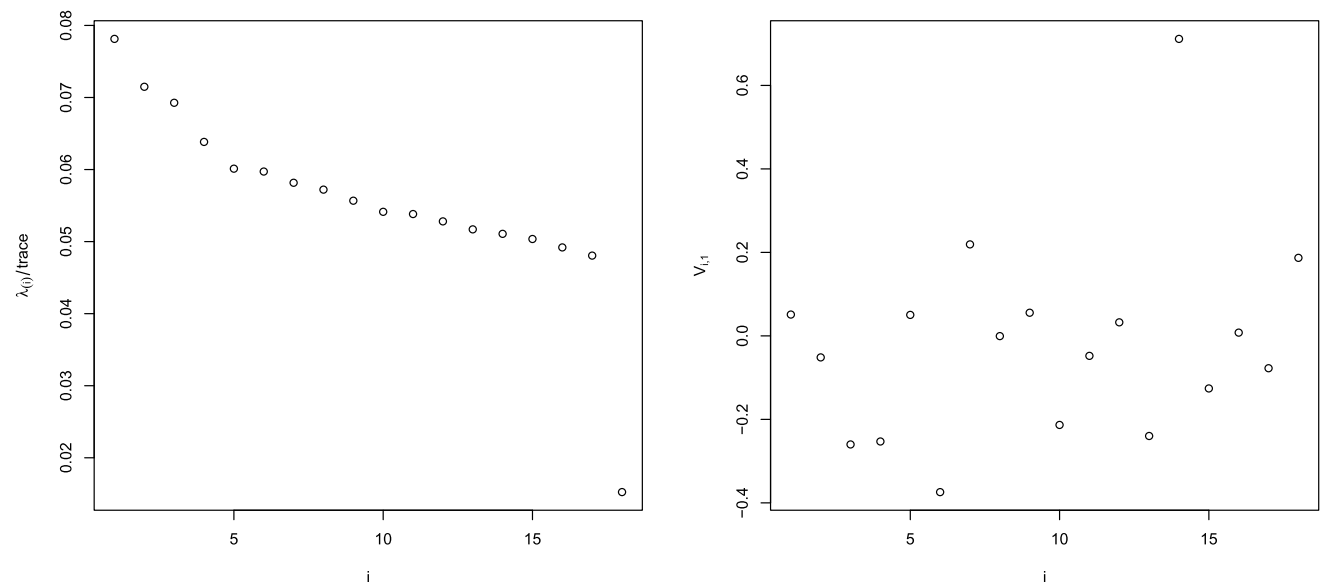

(d)

Figure 2. (Continued.)

ure 2(b) shows the normalized eigenvalues and the first unit eigenvector from a realization of this model. We notice a relatively large gap between the first and second eigenvalue and, in accordance with Section 3.4.4, we see that the first unit eigenvector is relatively close to a unit basis vector. Figure 2(c) shows the corresponding realizations for the model (5.1) with a specification according to Example 4.8, that is, Exponential(3)-distributed i.i.d. $\eta_{i, t}$ (meaning that $\mathbb{P}\left(\eta_{i, t}>x\right)=\exp (-3 x), x \geq 0$, which implies $\alpha=3$ and $\left.\mathbb{E}\left[\mathrm{e}^{3 \eta}\right]=\infty\right)$ and i.i.d. standard Gaussian $Z_{i t}$. Compared to the first simulated model, we see a slower decay in the magnitude of the ordered eigenvalues and a more spread out first unit eigenvector. This observation illustrates that although the limit behavior of this model and the one analyzed before should be very similar (cf. Example 4.8), convergence to the prescribed limit appears slower for the heavy-tailed volatility 
sequence than for the heavy-tailed innovations. Finally, Figure 2(d) shows a simulation drawn from (5.1) where the $\eta_{i, t}$ are i.i.d. such that $\mathbb{P}\left(\eta_{i, t}>x\right) \sim x^{-2} \exp (-3 x), x \rightarrow \infty$, and the $Z_{i t}$ are i.i.d. standard Gaussian. Again, $\alpha=3$, but direct calculations show that the distribution of $\eta_{i, t}$ is convolution equivalent, that is, it satisfies (4.2) instead of (4.1). The graphs are in line with the analysis in Example 4.9 and illustrate a very spread out dominant eigenvector. We note that while none of the three very simple models analyzed in the simulations above is able to fully describe the behavior of the analyzed data, the two models with heavy-tailed volatility and light-tailed innovations are able to explain a non-concentrated first unit eigenvector of the sample covariance matrix and therefore non-negligible dependence between components as seen in the data.

\section{Appendix A: Some $\alpha$-stable limit theory}

In this paper, we make frequently use of Theorem 4.3 in Mikosch and Wintenberger [39] which we quote for convenience:

Theorem A.1. Let $\left(\mathbf{Y}_{t}\right)$ be an $\mathbb{R}^{p}$-valued strictly stationary sequence, $\mathbf{S}_{n}=\mathbf{Y}_{1}+\cdots+\mathbf{Y}_{n}$ and $\left(a_{n}\right)$ be such that $n \mathbb{P}\left(\|\mathbf{Y}\|>a_{n}\right) \rightarrow 1$. Also write for $\varepsilon>0, \overline{\mathbf{Y}}_{t}=\mathbf{Y}_{t} \mathbf{1}\left(\left\|\mathbf{Y}_{t}\right\| \leq \varepsilon a_{n}\right), \underline{\mathbf{Y}}_{t}=\mathbf{Y}_{t}-\overline{\mathbf{Y}}_{t}$ and

$$
\overline{\mathbf{S}}_{l, n}=\sum_{t=1}^{l} \overline{\mathbf{Y}}_{t}, \quad \underline{\mathbf{S}}_{l, n}=\sum_{t=1}^{l} \underline{\mathbf{Y}}_{t}
$$

Assume the following conditions:

1. $\left(\mathbf{Y}_{t}\right)$ is regularly varying with index $\alpha \in(0,2) \backslash\{1\}$ and spectral tail process $\left(\boldsymbol{\Theta}_{j}\right)$.

2. A mixing condition holds: there exists an integer sequence $m_{n} \rightarrow \infty$ such that $k_{n}=$ $\left[n / m_{n}\right] \rightarrow \infty$ and

$$
\mathbb{E} \mathrm{e}^{i \mathbf{t}^{\prime} \underline{\mathbf{S}}_{n} / a_{n}}-\left(\mathbb{E} \mathrm{e}^{i \mathbf{t}^{\prime} \underline{\mathbf{S}}_{m_{n}, n} / a_{n}}\right)^{k_{n}} \rightarrow 0, \quad n \rightarrow \infty, \mathbf{t} \in \mathbb{R}^{p} .
$$

3. An anti-clustering condition holds:

$$
\lim _{l \rightarrow \infty} \limsup _{n \rightarrow \infty} \mathbb{P}\left(\max _{t=l, \ldots, m_{n}}\left\|\mathbf{Y}_{t}\right\|>\delta a_{n} \mid\left\|\mathbf{Y}_{0}\right\|>\delta a_{n}\right)=0, \quad \delta>0
$$

for the same sequence $\left(m_{n}\right)$ as in (2).

4. If $\alpha \in(1,2)$, in addition $\mathbb{E}[\mathbf{Y}]=\mathbf{0}$ and the vanishing small values condition holds:

$$
\lim _{\varepsilon \downarrow 0} \limsup _{n \rightarrow \infty} \mathbb{P}\left(a_{n}^{-1}\left\|\overline{\mathbf{S}}_{n}-\mathbb{E}\left[\overline{\mathbf{S}}_{n}\right]\right\|>\delta\right)=0, \quad \delta>0
$$

and $\sum_{i=1}^{\infty} \mathbb{E}\left[\left\|\boldsymbol{\Theta}_{i}\right\|\right]<\infty$.

Then $a_{n}^{-1} \mathbf{S}_{n} \stackrel{d}{\rightarrow} \xi_{\alpha}$ for an $\alpha$-stable $\mathbb{R}^{p}$-valued vector $\xi_{\alpha}$ with log-characteristic function

$$
\int_{0}^{\infty} \mathbb{E}\left[\mathrm{e}^{i y \mathbf{t}^{\prime} \sum_{j=0}^{\infty} \boldsymbol{\Theta}_{j}}-\mathrm{e}^{i y \mathbf{t}^{\prime} \sum_{j=1}^{\infty} \boldsymbol{\Theta}_{j}}-i y \mathbf{t}^{\prime} \mathbf{1}_{(1,2)}(\alpha)\right] d\left(-y^{\alpha}\right), \quad \mathbf{t} \in \mathbb{R}^{p} .
$$


Remark A.2. If we additionally assume that $\mathbf{Y}$ is symmetric, which implies $\mathbb{E}[\overline{\mathbf{Y}}]=\mathbf{0}$, then the statement of the theorem also holds for $\alpha=1$.

\section{Appendix B: (Joint) Tail behavior for products of regularly varying random variables}

In this paper, we make frequently use of the tail behavior of products of non-negative independent random variables $X$ and $Y$. In particular, we are interested in conditions for the existence of the limit

$$
\lim _{x \rightarrow \infty} \frac{\mathbb{P}(X Y>x)}{\mathbb{P}(X>x)}=q
$$

for some $q \in[0, \infty]$. We quote some of these results for convenience.

Lemma B.1. Let $X$ and $Y$ be independent random variables.

1. If $X$ and $Y$ are regularly varying with index $\alpha>0$, then $X Y$ is regularly varying with the same index.

2. If $X$ is regularly varying with index $\alpha>0$ and $\mathbb{E}\left[Y^{\alpha+\varepsilon}\right]<\infty$ for some $\varepsilon>0$, then (B.1) holds with $q=\mathbb{E}\left[Y^{\alpha}\right]$.

3. If $X$ and $Y$ are i.i.d. regularly varying with index $\alpha>0$ and $\mathbb{E}\left[Y^{\alpha}\right]<\infty$, then (B.1) holds with $q=2 \mathbb{E}\left[|Y|^{\alpha}\right]$ iff

$$
\lim _{M \rightarrow \infty} \limsup _{x \rightarrow \infty} \frac{\mathbb{P}(X Y>x, M<Y \leq x / M)}{\mathbb{P}(X>x)}=0 .
$$

4. If $X$ and $Y$ are regularly varying with index $\alpha>0, \mathbb{E}\left[Y^{\alpha}+X^{\alpha}\right]<\infty, \lim _{x \rightarrow \infty} \mathbb{P}(Y>$ $x) / \mathbb{P}(X>x)=0$ and (B.2) holds, then (B.1) holds with $q=\mathbb{E}\left[|Y|^{\alpha}\right]$.

5. Assume that $\mathbb{E}\left[|Y|^{\alpha}\right]=\infty$. Then (B.1) holds with $q=\infty$.

Proof. (1) This is proved in Embrechts and Goldie [28].

(2) This is Breiman's [11] result.

(3) This is Proposition 3.1 in Davis and Resnick [15].

(4) This part is proved similarly to (3); we borrow the ideas from [15]. For $M>0$, we have the following decomposition

$$
\begin{aligned}
\frac{\mathbb{P}(X Y>x)}{\mathbb{P}(X>x)} & =\frac{\mathbb{P}(X Y>x, Y \leq M)}{\mathbb{P}(X>x)}+\frac{\mathbb{P}(X Y>x, M<Y \leq x / M)}{\mathbb{P}(X>x)}+\frac{\mathbb{P}(X Y>x, Y>x / M)}{\mathbb{P}(X>x)} \\
& \sim \mathbb{E}\left[Y^{\alpha} \mathbf{1}(Y \leq M)\right]+\frac{\mathbb{P}(X Y>x, M<Y \leq x / M)}{\mathbb{P}(X>x)}+\mathbb{E}\left[(X \wedge M)^{\alpha}\right] \frac{\mathbb{P}(Y>x)}{\mathbb{P}(X>x)} \\
& =\mathbb{E}\left[Y^{\alpha} \mathbf{1}(Y \leq M)\right]+\frac{\mathbb{P}(X Y>x, M<Y \leq x / M)}{\mathbb{P}(X>x)}+o(1) .
\end{aligned}
$$


Here we applied Breiman's result twice. The second term vanishes by virtue of (B.2). Thus $q=\mathbb{E}\left[Y^{\alpha}\right]$.

(5) The same argument as for (4) yields as $x \rightarrow \infty$,

$$
\frac{\mathbb{P}(X Y>x)}{\mathbb{P}(X>x)} \geq \frac{\mathbb{P}(X Y>x, Y \leq M)}{\mathbb{P}(X>x)} \sim \mathbb{E}\left[Y^{\alpha} \mathbf{1}(Y \leq M)\right] .
$$

Then (B.1) with $q=\infty$ is immediate.

Lemma B.2. Let $Y_{1}, \ldots, Y_{p} \geq 0$ be i.i.d. regularly varying random variables with index $\alpha>0$. Assume that

$$
\lim _{t \rightarrow \infty} \frac{\mathbb{P}\left(Y_{1} \cdot Y_{2}>t\right)}{\mathbb{P}\left(Y_{1}>t\right)}=c \in(0, \infty) .
$$

Then for any $a_{1}, \ldots, a_{p} \geq 0$ such that $a_{\max }:=\max _{j=1, \ldots, p} a_{j}>0$ and any $v>0$ we have

$$
\lim _{t \rightarrow \infty} \frac{\mathbb{P}\left(\prod_{i=1}^{p} Y_{i}^{a_{i}}>v t\right)}{\mathbb{P}\left(Y_{1}^{a_{\max }}>t\right)}=\sum_{j: a_{j}=a_{\max }} \lim _{s \rightarrow 0} \lim _{t \rightarrow \infty} \frac{\mathbb{P}\left(\prod_{i=1}^{p} Y_{i}^{a_{i}}>v t, Y_{j}^{a_{\max }}>s t\right)}{\mathbb{P}\left(Y_{1}^{a_{\max }}>t\right)}
$$

and

$$
\lim _{s \rightarrow 0} \limsup _{t \rightarrow \infty} \frac{\mathbb{P}\left(\prod_{i=1}^{p} Y_{i}^{a_{i}}>v t, \max _{j=1, \ldots, p} Y_{j}^{a_{\max }} \leq s t\right)}{\mathbb{P}\left(Y_{1}^{a_{\max }}>t\right)}=0 .
$$

Proof. In view of Davis and Resnick [16], the only possible value for $c$ in (B.3) is $2 \mathbb{E}\left[Y_{1}^{\alpha}\right]$ (which implies that $\mathbb{E}\left[Y_{1}^{\alpha}\right]<\infty$ ). Furthermore, we note that the product $\prod_{j: a_{j}=a_{\max }} Y_{j}^{a_{j}}$ is regularly varying with index $-\alpha / a_{\max }$; see Embrechts and Goldie [28], Corollary on page 245. By Breiman's lemma, this implies that

$$
\begin{aligned}
& \lim _{t \rightarrow \infty} \frac{\mathbb{P}\left(\prod_{i=1}^{p} Y_{i}^{a_{i}}>v t\right)}{\mathbb{P}\left(Y_{1}^{a_{\max }}>t\right)} \\
& =\lim _{t \rightarrow \infty} \frac{\mathbb{P}\left(Y_{1}^{a_{\max }}>v t\right)}{\mathbb{P}\left(Y_{1}^{a_{\max }}>t\right)} \frac{\mathbb{P}\left(\prod_{i=1}^{p} Y_{i}^{a_{i}}>v t\right)}{\mathbb{P}\left(Y_{1}^{a_{\max }}>v t\right)} \\
& =v^{-\alpha / a_{\max }}\left(\prod_{j: a_{j} \neq a_{\max }} \mathbb{E}\left[Y_{j}^{\alpha a_{j} / a_{\max }}\right]\right) \lim _{t \rightarrow \infty} \frac{\mathbb{P}\left(\prod_{j: a_{j}=a_{\max }} Y_{j}^{a_{\max }}>v t\right)}{\mathbb{P}\left(Y_{1}^{a_{\max }}>v t\right)} .
\end{aligned}
$$

By Lemma 2.5 in Embrechts and Goldie [29] (cf. also Chover, Ney and Wainger [13]) this equals

$$
v^{-\alpha / a_{\max }}\left(\prod_{j: a_{j} \neq a_{\max }} \mathbb{E}\left[Y_{j}^{\alpha a_{j} / a_{\max }}\right]\right)\left|\left\{j: a_{j}=a_{\max }\right\}\right| \mathbb{E}\left[Y_{1}^{\alpha}\right]^{\left|\left\{j: a_{j}=a_{\max }\right\}\right|-1} .
$$


On the other hand, we have

$$
\begin{aligned}
& \sum_{j: a_{j}=a_{\max }} \lim _{s \rightarrow 0} \lim _{t \rightarrow \infty} \frac{\mathbb{P}\left(\prod_{i=1}^{p} Y_{i}^{a_{i}}>v t, Y_{j}^{a_{\max }}>s t\right)}{\mathbb{P}\left(Y_{1}^{a_{\max }}>t\right)} \\
& =\sum_{j: a_{j}=a_{\max }} \lim _{s \rightarrow 0} \lim _{t \rightarrow \infty} \frac{\mathbb{P}\left(Y_{j}^{a_{\max }} \min \left(s^{-1}, v^{-1} \prod_{k \neq j} Y_{k}^{a_{k}}\right)>t\right)}{\mathbb{P}\left(Y_{j}^{a_{\max }}>t\right)} \\
& =\sum_{j: a_{j}=a_{\max }} \lim _{s \rightarrow 0} \mathbb{E}\left[\left(\min \left(s^{-1}, v^{-1} \prod_{k \neq j} Y_{k}^{a_{k}}\right)\right)^{\alpha / a_{\max }}\right] \\
& =v^{-\alpha / a_{\max }} \sum_{j: a_{j}=a_{\max }} \prod_{k \neq j} \mathbb{E}\left[Y_{k}^{\alpha a_{k} / a_{\max }}\right] \\
& =v^{-\alpha / a_{\max }}\left(\prod_{j: a_{j} \neq a_{\max }} \mathbb{E}\left[Y_{j}^{\alpha a_{j} / a_{\max }}\right]\right)\left|\left\{j: a_{j}=a_{\max }\right\}\right| \mathbb{E}\left[Y_{1}^{\alpha}\right]^{\left|\left\{j: a_{j}=a_{\max }\right\}\right|-1},
\end{aligned}
$$

where we applied Breiman's lemma in the second step to the bounded random variable $\min \left(s^{-1}\right.$, $\left.v^{-1} \prod_{k \neq j} Y_{k}^{a_{k}}\right)$, and the monotone convergence theorem in the penultimate step. This proves (B.4). To prove (B.5) note that for $s>0$,

$$
\begin{aligned}
\frac{\mathbb{P}\left(\prod_{i=1}^{p} Y_{i}^{a_{i}}>v t\right)}{\mathbb{P}\left(Y_{1}^{a_{\max }}>t\right)} \geq & \frac{\mathbb{P}\left(\prod_{i=1}^{p} Y_{i}^{a_{i}}>v t, \max _{j=1, \ldots, p} Y_{j}^{a_{\max }} \leq s t\right)}{\mathbb{P}\left(Y_{1}^{a_{\max }}>t\right)} \\
& +\sum_{j: a_{j}=a_{\max }} \frac{\mathbb{P}\left(\prod_{i=1}^{p} Y_{i}^{a_{i}}>v t, Y_{j}^{a_{\max }}>s t\right)}{\mathbb{P}\left(Y_{1}^{a_{\max }}>t\right)} \\
& -\frac{\mathbb{P}\left(\prod_{i=1}^{p} Y_{i}^{a_{i}}>v t, Y_{j_{1}}^{a_{\max }}>s t, Y_{j 2}^{a_{\max }}>s t \text { for some } j_{1} \neq j_{2}\right)}{\mathbb{P}\left(Y_{1}^{a_{\max }}>t\right)}, \quad s>0 .
\end{aligned}
$$

The last summand on the right-hand side converges to 0 as $t \rightarrow \infty$ by independence of the $Y_{j}^{\prime} s$. Moreover, the left-hand term and the second term on the right-hand side become equal by first $t \rightarrow \infty$ and then $s \rightarrow 0$, in view of (B.4). Therefore the first right-hand term vanishes by first $t \rightarrow \infty$ and then $s \rightarrow 0$. This proves the statement.

Proposition B.3. Let $Y_{1}, \ldots, Y_{p} \geq 0$ be i.i.d. regularly varying with index $\alpha$ and $\left(a_{i j}\right) \in$ $[0, \infty)^{n \times p}, n, p \geq 1$, be such that $\max _{1 \leq i \leq n} a_{i k}=a_{\max }:=\max _{i, j} a_{i j}>0$ for any $1 \leq k \leq p$.

(i) Assume that (B.3) holds. Then the random vector

$$
\mathbf{Y}:=\left(\prod_{j=1}^{p} Y_{j}^{a_{i j}}\right)_{1 \leq i \leq n}
$$


is regularly varying with index $\alpha / a_{\max }$. Furthermore, up to a constant the limit measure $\mu$ of $\mathbf{Y}$ is given by $\sum_{j=1}^{p} \mu_{j}$, where for any Borel set $B \in[0, \infty]^{n}$ bounded away from $\mathbf{0}$ and $v_{\alpha}(d z)=$ $\alpha z^{-\alpha-1} d z$,

$$
\mu_{j}(B)=\int_{0}^{\infty} \mathbb{P}\left(\left(\mathbf{1}\left(a_{i j}=a_{\max }\right) z^{a_{\max }} \prod_{k \neq j} Y_{k}^{a_{i k}}\right)_{1 \leq i \leq n} \in B\right) v_{\alpha}(d z) .
$$

(ii) Assume that $\mathbb{E}\left[Y_{1}^{\alpha}\right]=\infty$. Set

$$
\begin{aligned}
& p_{\text {eff }}:=\max _{i}\left|\left\{1 \leq j \leq p: a_{i j}=a_{\max }\right\}\right|, \\
& P_{\text {eff }}:=\left\{A \subset\{1, \ldots, p\}:|A|=p_{\text {eff }} \wedge \exists i: \forall j \in A: a_{i j}=a_{\max }\right\} .
\end{aligned}
$$

Then the random vector $\mathbf{Y}$ in (B.6) is regularly varying with index $\alpha / a_{\max }$. Furthermore, up to a constant the limit measure $\mu$ of $\mathbf{Y}$ is equal to $\sum_{A \in P_{\mathrm{eff}}} \mu_{A}$, where for any Borel set $B \in[0, \infty]^{n}$ bounded away from $\mathbf{0}$,

$$
\mu_{A}(B)=\int_{0}^{\infty} \mathbb{P}\left(\left(\mathbf{1}\left(a_{i j}=a_{\max } \forall j \in A\right) z^{a_{\max }} \prod_{k \notin A} Y_{k}^{a_{i k}}\right)_{1 \leq i \leq n} \in B\right) v_{\alpha}(d z) .
$$

Proof. (i) Let $B \in[0, \infty]^{n}$ be a Borel set bounded away from $\mathbf{0}$. For $s>0$ we have

$$
\begin{aligned}
\frac{\mathbb{P}(\mathbf{Y} \in t B)}{\mathbb{P}\left(Y_{1}^{a_{\max }}>t\right)}= & \frac{\mathbb{P}\left(\mathbf{Y} \in t B, \max _{j=1, \ldots, p} Y_{j}^{a_{\max }} \leq s t\right)}{\mathbb{P}\left(Y_{1}^{a_{\max }}>t\right)}+\sum_{j=1}^{p} \frac{\mathbb{P}\left(\mathbf{Y} \in t B, Y_{j}^{a_{\max }}>s t\right)}{\mathbb{P}\left(Y_{1}^{a_{\max }}>t\right)} \\
& -\frac{\mathbb{P}\left(\mathbf{Y} \in t B, Y_{j_{1}}^{a_{\max }}>s t, Y_{j_{2}}^{a_{\max }}>s t, \text { for some } j_{1} \neq j_{2}\right)}{\mathbb{P}\left(Y_{1}^{a_{\max }}>t\right)} .
\end{aligned}
$$

Since $B$ is bounded away from $\mathbf{0}$, there exists $v>0$ and $1 \leq i \leq n$ such that $B \subset\left\{\left(x_{1}, \ldots, x_{n}\right) \in\right.$ $\left.[0, \infty]^{n}: x_{i}>v\right\}$. From Lemma B.2, (B.5) the first summand in (B.9) therefore tends to 0 by first $t \rightarrow \infty$ and then $s \rightarrow 0$. Furthermore, the third summand converges to zero as $t \rightarrow \infty$ by independence of the $Y_{j}^{\prime} s$. We are thus left to show

$$
\lim _{s \searrow 0} \lim _{t \rightarrow \infty} \frac{\mathbb{P}\left(\mathbf{Y} \in t B, Y_{j}^{a_{\max }}>s t\right)}{\mathbb{P}\left(Y_{1}^{a_{\max }}>t\right)}=\mu_{j}(B), 1 \leq j \leq p,
$$

with $\mu_{j}$ as in (B.7). For $s>0$ write

$$
\begin{aligned}
\lim _{t \rightarrow \infty} \frac{\mathbb{P}\left(\mathbf{Y} \in t B, Y_{j}^{a_{\max }}>s t\right)}{\mathbb{P}\left(Y_{1}^{a_{\max }}>t\right)} \\
=s^{-\alpha / a_{\max }} \lim _{t \rightarrow \infty} \mathbb{P}\left(\mathbf{Y} \in t B \mid Y_{j}^{a_{\max }}>s t\right)
\end{aligned}
$$




$$
\begin{aligned}
& =s^{-\alpha / a_{\max }} \lim _{t \rightarrow \infty} \mathbb{P}\left(\left(\left(\frac{Y_{j}^{a_{\max }}}{s t}\right)^{\frac{a_{i j}}{a_{\max }}} s^{\frac{a_{i j}}{a_{\max }}} t^{\frac{a_{i j}}{a_{\max }}}-1 \prod_{k \neq j} Y_{k}^{a_{i k}}\right)_{1 \leq i \leq n} \in B \mid Y_{j}^{a_{\max }}>s t\right) \\
& =s^{-\alpha / a_{\max }} \int_{1}^{\infty} \mathbb{P}\left(\left(\mathbf{1}\left(a_{i j}=a_{\max }\right) s y \prod_{k \neq j} Y_{k}^{a_{i k}}\right)_{1 \leq i \leq n} \in B\right) v_{\alpha / a_{\max }}(d y) .
\end{aligned}
$$

Substituting $s y$ by $z$ in the integral finally gives

$$
\lim _{s \searrow 0} \lim _{t \rightarrow \infty} \frac{\mathbb{P}\left(\mathbf{Y} \in t B, Y_{j}^{a_{\max }}>s t\right)}{\mathbb{P}\left(Y_{1}^{a_{\max }}>t\right)}=\int_{0}^{\infty} \mathbb{P}\left(\left(\mathbf{1}\left(a_{i j}=a_{\max }\right) z^{a_{\max }} \prod_{k \neq j} Y_{k}^{a_{i k}}\right)_{1 \leq i \leq n} \in B\right) v_{\alpha}(d z) .
$$

(ii) Note first that under our assumptions for any $1 \leq n_{1}<n_{2} \leq p$,

$$
\begin{aligned}
\lim _{t \rightarrow \infty} \frac{\mathbb{P}\left(\prod_{j=1}^{n_{2}} Y_{j}>t\right)}{\mathbb{P}\left(\prod_{j=1}^{n_{1}} Y_{j}>t\right)} & =\lim _{t \rightarrow \infty} \int_{0}^{\infty} \frac{\mathbb{P}\left(\prod_{j=1}^{n_{1}} Y_{j}>t / y\right)}{\mathbb{P}\left(\prod_{j=1}^{n_{1}} Y_{j}>t\right)} P^{\prod_{j=n_{1}+1}^{n_{2}} Y_{j}}(d y) \\
& \geq \mathbb{E}\left[\prod_{j=n_{1}+1}^{n_{2}} Y_{j}^{\alpha}\right]=\infty
\end{aligned}
$$

by Fatou's lemma and the regular variation of $\prod_{j=1}^{n_{1}} Y_{j}$. Write now

$$
\mathbf{Y}=\sum_{\substack{1 \leq i \leq n \\\left\{\left\{j: a_{i j}=a_{\max }\right\} \mid=p_{\mathrm{eff}}\right.}} \prod_{j=1}^{p} Y_{j}^{a_{i j}} \mathbf{e}_{i}+\sum_{\substack{1 \leq i \leq n \\\left|\left\{j: a_{i j}=a_{\max }\right\}\right|<p_{\mathrm{eff}}}} \prod_{j=1}^{p} Y_{j}^{a_{i j}} \mathbf{e}_{i},
$$

where $\mathbf{e}_{i}$ stands for the $i$ th unit vector. The first sum can also be written as

$$
\sum_{A \in P_{\mathrm{eff}}} \operatorname{diag}\left(\left(\mathbf{1}\left(a_{i j}=a_{\max } \forall j \in A\right) \prod_{k \notin A} Y_{k}^{a_{i k}}\right)_{1 \leq i \leq n}\right) \prod_{j \in A} Y_{j}^{a_{\max }}=: \sum_{A \in P_{\mathrm{eff}}} \mathbf{Y}^{A},
$$

where for each summand the random matrix and the random factor are independent and for the non-zero entries of the matrix we have $a_{i k}<a_{\max }$ since $k \notin A$. Thus, by the multivariate version of Breiman's lemma each $\mathbf{Y}^{A}$ is a multivariate regularly varying vector with limit measure $\mu_{A}$ (up to a constant multiplier) as in (B.8) and normalizing function $P\left(\prod_{i=1}^{p_{\text {eff }}} Y_{i}^{a_{\max }}>x\right)$. Furthermore, for $A, A^{\prime} \in P_{\text {eff }}$ with $A \neq A^{\prime}$ and $i, i^{\prime}$ such that $a_{i j}=a_{\max } \forall j \in A$ and $a_{i^{\prime} j}=a_{\max } \forall j \in A^{\prime}$ we have

$$
\begin{aligned}
& \frac{\mathbb{P}\left(\mathbf{Y}_{i}^{A}>x, \mathbf{Y}_{i^{\prime}}^{A^{\prime}}>x\right)}{\mathbb{P}\left(\prod_{i=1}^{p_{\text {eff }}} Y_{i}^{a_{\max }}>x\right)} \\
& \quad=\frac{\mathbb{P}\left(\left(\prod_{j \in A \cap A^{\prime}} Y_{j}\right)^{a_{\max }} \prod_{j \in\left(A \cap A^{\prime}\right)^{c}} Y_{j}^{a_{i j}}>x,\left(\prod_{j \in A \cap A^{\prime}} Y_{j}\right)^{a_{\max }} \prod_{j \in\left(A \cap A^{\prime}\right)^{c}} Y_{j}^{a_{i^{\prime} j}}>x\right)}{\mathbb{P}\left(\prod_{i=1}^{p_{\text {eff }}} Y_{i}^{a_{\max }}>x\right)} .
\end{aligned}
$$


By Janßen and Drees [34], Theorem 4.2 (in connection with Remark 4.3(ii) and the minor change that our random variables are regularly varying with index $\alpha$ instead of 1$)$, the numerator behaves asymptotically like $\mathbb{P}\left(\left(\prod_{j \in A \cap A^{\prime}} Y_{j}\right)^{a_{\max }}>x\right)$, since $\kappa_{0}=a_{\max }^{-1}, \kappa_{j}=0, j \in\left(A \cap A^{\prime}\right)^{c}$ is the unique non-negative optimal solution to

$$
\kappa_{0}+\sum_{j \in\left(A \cap A^{\prime}\right)^{c}} \kappa_{j} \rightarrow \min !
$$

under

$$
\kappa_{0} a_{\max }+\sum_{j \in\left(A \cap A^{\prime}\right)^{c}} \kappa_{j} a_{i j} \geq 1, \quad \kappa_{0} a_{\max }+\sum_{j \in\left(A \cap A^{\prime}\right)^{c}} \kappa_{j} a_{i^{\prime} j} \geq 1 .
$$

This is because $\min \left(a_{i j}, a_{i^{\prime} j}\right)<a_{\max }$ and $\max \left(a_{i j}, a_{i^{\prime} j}\right) \leq a_{\max }$ for all $j \in\left(A \cap A^{\prime}\right)^{c}$. Since $A \neq A^{\prime}$, we have $\left|A \cap A^{\prime}\right|<p_{\text {eff }}$ and thus, by (B.10), the expression (B.13) converges to 0 as $x \rightarrow$ $\infty$. Therefore, each component of $\mathbf{Y}^{A}$ is asymptotically independent of each component of $\mathbf{Y}^{A^{\prime}}$ and thus the sum in (B.12) is multivariate regularly varying with limit measure $\sum_{A \in P_{\text {eff }}} \mu_{A}$ and normalizing function $\mathbb{P}\left(\prod_{i=1}^{p_{\text {eff }}} Y_{i}^{a_{\max }}>x\right)$. Since the second sum in (B.11) consists by (B.10) only of random vectors for which $\mathbb{P}\left(\left\|\prod_{j=1}^{p} Y_{j}^{a_{i j}} \mathbf{e}_{i}\right\|>x\right)=\mathbb{P}\left(\prod_{j=1}^{p} Y_{j}^{a_{i j}}>x\right)=o\left(\mathbb{P}\left(\prod_{i=1}^{p_{\text {eff }}} Y_{i}^{a_{\max }}>\right.\right.$ $x)$ ), we have that $\mathbf{Y}$ is regularly varying with index $\alpha / a_{\max }$ and limit measure $\sum_{A \in P_{\text {eff }}} \mu_{A}$ by Lemma 3.12 in Jessen and Mikosch [35].

\section{Acknowledgements}

Thomas Mikosch's and Xiaolei Xie's research is partly supported by the Danish Research Council Grant DFF-4002-00435 "Large random matrices with heavy tails and dependence". Parts of the paper were written when Mohsen Rezapour visited the Department of Mathematics at the University of Copenhagen December 2015-January 2016. He would like to thank the Department of Mathematics for hospitality. We would like to thank the reviewers of our paper for careful reading and constructive comments.

\section{References}

[1] Andersen, T.G., Davis, R.A., Kreiss, J.-P. and Mikosch, T., eds. (2009). Handbook of Financial Time Series. Berlin: Springer.

[2] Auffinger, A., Ben Arous, G. and Péché, S. (2009). Poisson convergence for the largest eigenvalues of heavy tailed random matrices. Ann. Inst. Henri Poincaré Probab. Stat. 45 589-610. MR2548495

[3] Bai, Z.D. and Yin, Y.Q. (1993). Limit of the smallest eigenvalue of a large-dimensional sample covariance matrix. Ann. Probab. 21 1275-1294. MR1235416

[4] Basrak, B., Davis, R.A. and Mikosch, T. (2002). A characterization of multivariate regular variation. Ann. Appl. Probab. 12 908-920. MR1925445

[5] Basrak, B. and Segers, J. (2009). Regularly varying multivariate time series. Stochastic Process. Appl. 119 1055-1080. MR2508565 
[6] Belinschi, S., Dembo, A. and Guionnet, A. (2009). Spectral measure of heavy tailed band and covariance random matrices. Comm. Math. Phys. 289 1023-1055. MR2511659

[7] Benaych-Georges, F. and Péché, S. (2014). Localization and delocalization for heavy tailed band matrices. Ann. Inst. Henri Poincaré Probab. Stat. 50 1385-1403. MR3269999

[8] Ben Arous, G. and Guionnet, A. (2008). The spectrum of heavy tailed random matrices. Comm. Math. Phys. 278 715-751. MR2373441

[9] Bhatia, R. (1997). Matrix Analysis. Graduate Texts in Mathematics 169. New York: Springer. MR1477662

[10] Bordenave, C. and Guionnet, A. (2013). Localization and delocalization of eigenvectors for heavytailed random matrices. Probab. Theory Related Fields 157 885-953. MR3129806

[11] Breiman, L. (1965). On some limit theorems similar to the arc-sin law. Theory Probab. Appl. 10 323-331.

[12] Campbell, J., Lo, A.W. and MacKinlay, A.C. (1997). The Econometrics of Financial Markets. Princeton: Princeton Univ. Press.

[13] Chover, J., Ney, P. and Wainger, S. (1973). Functions of probability measures. J. Anal. Math. 26 255302. MR0348393

[14] Cline, D.B.H. (1986). Convolution tails, product tails and domains of attraction. Probab. Theory Related Fields 72 529-557. MR0847385

[15] Davis, R.A. and Resnick, S. (1985). More limit theory for the sample correlation function of moving averages. Stochastic Process. Appl. 20 257-279. MR0808161

[16] Davis, R.A. and Resnick, S. (1986). Limit theory for the sample covariance and correlation functions of moving averages. Ann. Statist. 14 533-558. MR0840513

[17] Davis, R.A., Heiny, J., Mikosch, T. and Xie, X. (2016). Extreme value analysis for the sample autocovariance matrices of heavy-tailed multivariate time series. Extremes 19 517-547. MR3535965

[18] Davis, R.A. and Hsing, T. (1995). Point process and partial sum convergence for weakly dependent random variables with infinite variance. Ann. Probab. 23 879-917. MR1334176

[19] Davis, R.A. and Mikosch, T. (2000). The sample autocorrelations of financial time series models. In Nonlinear and Nonstationary Signal Processing (Cambridge, 1998) 247-274. Cambridge: Cambridge Univ. Press. MR1831322

[20] Davis, R.A. and Mikosch, T. (2001). Point process convergence of stochastic volatility processes with application to sample autocorrelation. J. Appl. Probab. 38A 93-104. MR1915537

[21] Davis, R.A. and Mikosch, T. (2009). Probabilistic properties of stochastic volatility models. In Handbook of Financial Time Series (T.G. Andersen, R.A. Davis, J.-P. Kreiss and T. Mikosch, eds.) 255-268. Berlin: Springer. 2009.

[22] Davis, R.A. and Mikosch, T. (2009). The extremogram: A correlogram for extreme events. Bernoulli 15 977-1009. MR2597580

[23] Davis, R.A., Mikosch, T. and Pfaffel, O. (2016). Asymptotic theory for the sample covariance matrix of a heavy-tailed multivariate time series. Stochastic Process. Appl. 126 767-799. MR3452812

[24] Davis, R.A., Pfaffel, O. and Stelzer, R. (2014). Limit theory for the largest eigenvalues of sample covariance matrices with heavy-tails. Stochastic Process. Appl. 124 18-50. MR3131285

[25] Ding, X. and Yang, F. (2016). A necessary and sufficient condition for edge universality at the largest singular values of covariance matrices. Preprint. Available at arXiv:1607.06873.

[26] Doukhan, P. (1994). Mixing: Properties and Examples. Lecture Notes in Statistics 85. Springer: Berlin. MR1312160

[27] Drees, H. (2003). Extreme quantile estimation for dependent data, with applications to finance. Bernoulli 9 617-657. MR1996273

[28] Embrechts, P. and Goldie, C.M. (1980). On closure and factorization properties of subexponential and related distributions. J. Aust. Math. Soc. A 29 243-256. MR0566289 
[29] Embrechts, P. and Goldie, C.M. (1982). On convolution tails. Stochastic Process. Appl. 13 263-278. MR0671036

[30] Feller, W. (1971). An Introduction to Probability Theory and Its Applications. Vol. II., 2nd ed. New York: Wiley. MR0270403

[31] Heiny, J. and Mikosch, T. (2016). Eigenvalues and eigenvectors of heavy-tailed sample covariance matrices with general growth rates: The iid case. Technical report.

[32] Hult, H. and Lindskog, F. (2005). Extremal behavior of regularly varying stochastic processes. Stochastic Process. Appl. 115 249-274. MR2111194

[33] Hult, H. and Lindskog, F. (2006). Regular variation for measures on metric spaces. Publ. Inst. Math. (Beograd) (N.S.) 80(94) 121-140. MR2281910

[34] Janssen, A. and Drees, H. (2016). A stochastic volatility model with flexible extremal dependence structure. Bernoulli 22 1448-1490. MR3474822

[35] Jessen, A.H. and Mikosch, T. (2006). Regularly varying functions. Publ. Inst. Math. (Beograd) (N.S.) 80(94) 171-192. MR2281913

[36] Kulik, R. and Soulier, P. (2015). Heavy tailed time series with extremal independence. Extremes 18 273-299. MR3351817

[37] Lam, C. and Yao, Q. (2012). Factor modeling for high-dimensional time series: Inference for the number of factors. Ann. Statist. 40 694-726. MR2933663

[38] Mikosch, T. and Rezapour, M. (2013). Stochastic volatility models with possible extremal clustering. Bernoulli 19 1688-1713. MR3129030

[39] Mikosch, T. and Wintenberger, O. (2016). A large deviations approach to limit theory for heavy-tailed time series. Probab. Theory Related Fields 166 233-269. MR3547739

[40] Petrov, V.V. (1995). Limit Theorems of Probability Theory. Oxford Studies in Probability 4. New York: Oxford Univ. Press. MR1353441

[41] Plerou, V., Gopikrishnan, P., Rosenow, B., Amaral, L.A.N., Guhr, T. and Stanley, H.E. (2002). Random matrix approach to cross correlations in financial data. Phys. Rev. E $\mathbf{6 5} 066126$.

[42] Resnick, S.I. (1987). Extreme Values, Regular Variation, and Point Processes. New York: Springer. MR0900810

[43] Resnick, S.I. (2007). Heavy-Tail Phenomena: Probabilistic and Statistical Modeling. Springer Series in Operations Research and Financial Engineering. New York: Springer. MR2271424

[44] Rootzén, H. (1986). Extreme value theory for moving average processes. Ann. Probab. 14 612-652. MR0832027

[45] Soshnikov, A. (2004). Poisson statistics for the largest eigenvalues of Wigner random matrices with heavy tails. Electron. Commun. Probab. 9 82-91 (electronic). MR2081462

[46] Soshnikov, A. (2006). Poisson statistics for the largest eigenvalues in random matrix ensembles. In Mathematical Physics of Quantum Mechanics. Lecture Notes in Physics 690 351-364. Berlin: Springer. MR2234922

[47] Tao, T. and Vu, V. (2012). Random covariance matrices: Universality of local statistics of eigenvalues. Ann. Probab. 40 1285-1315. MR2962092

[48] Taylor, S.J. (1986). Modelling Financial Time Series. Chichester: Wiley.

[49] von Bahr, B. and Esseen, C.-G. (1965). Inequalities for the $r$ th absolute moment of a sum of random variables, $1 \leq r \leq 2$. Ann. Math. Stat. 36 299-303. MR0170407

Received May 2016 and revised September 2016 